\title{
Vibrations, Tunneling, and Transition Dipole Moments in the Water Dimer
}

\author{
Michael J. Smit, Gerrit C. Groenenboom, Paul E. S. Wormer, and Ad van der Avoird* \\ Institute of Theoretical Chemistry, NSR-Center, University of Nijmegen, \\ Toernooiveld, 6525 ED Nijmegen, The Netherlands
}

Robert Bukowski and Krzysztof Szalewicz

Department of Physics and Astronomy, University of Delaware, Newark, Delaware 19716, USA

Received: December 31, 2000

\begin{abstract}
The wave functions of the dimers $\left(\mathrm{H}_{2} \mathrm{O}\right)_{2}$ and $\left(\mathrm{D}_{2} \mathrm{O}\right)_{2}$ computed earlier [Groenenboom et al. J. Chem. Phys. 2000, 113, 6702] are analyzed. Their tunneling and vibrational behavior is inspected and compared with earlier experimental assignments of the tunneling and vibrational modes. The transition dipole moments between different vibration-rotation-tunneling states are computed, and suggestions are made for possible spectroscopic observations of new far-infrared lines. A group-theoretical analysis shows that only the states of $E^{ \pm}$symmetry of the water dimer have a permanent dipole moment. A model is presented which relates the values of the parallel transition dipole moments associated with tunneling between states of $A_{1}^{ \pm}$and $B_{1}^{ \pm}$symmetry and between states of $A_{2}^{ \pm}$and $B_{2}^{ \pm}$symmetry to the permanent dipole moment of the $E^{ \pm}$states.
\end{abstract}

\section{Introduction}

Liquid water is the most important biological substance. If extraterrestrial life resembles that on Earth it will definitely require liquid water. ${ }^{1}$ This is why the recent discovery ${ }^{2}$ of sources of liquid water beneath the surface of Mars has drawn worldwide attention. See refs 3 and 4 for further reviews of many different aspects of liquid water and see, for instance, ref 5 for a discussion - from a pure physicochemical point of viewof why water is such an intriguing fluid.

Despite the many years of study (see the recent work in refs $6-8)$, the quantitative statistical thermodynamical theory of liquid water and ice is still not complete. There are good reasons to believe that this is due to an insufficient knowledge of the pair and many-body interactions between water molecules. In computer simulations of water, it is common to use so-called effective water pair potentials in which the many-body interactions are implicitly included. However, ab initio calculations have shown ${ }^{9-16}$ that three-body effects are substantial and cannot accurately be accounted for by effective pair potentials. Very recently, Mahoney and Jorgensen ${ }^{17}$ obtained a new empirical pair potential: TIP5P. Although the temperature range of its applicability is much wider than that of any of the earlier effective pair potentials, even the TIP5P potential shows the limitation of such potentials. So, there is still quite some interest in the study of water pair potentials. The best testing ground for the quality of such potentials is formed by the spectra of the dimer.

In earlier papers, ${ }^{18,19}$ we discussed several representations of a water pair potential obtained from symmetry adapted perturbation theory (SAPT) calculations on the dimer. The best one ${ }^{19}$ is a site-site fit of the ab initio SAPT potential, called SAPT-5s, with eight sites per molecule, five of which are symmetrydistinct. It is based on rigid monomers in their vibrationally averaged ground-state geometry. In a computation of the

* To whom correspondence should be addressed. E-mail: avda@theochem kun.nl. Fax: +31 243653041. vibrational-rotational-tunneling (VRT) levels and spectra of the dimer ${ }^{20,21}$ this potential produced good agreement with the experimental data. Subsequently, we used one of the experimentally observed level splittings to tune some of the parameters in this potential, then referred to as SAPT-5st. Comparison with high-resolution microwave data ${ }^{22-27}$ and the extensive farinfrared data from the Berkeley group ${ }^{28-34}$ has shown that this SAPT-5st potential is far better in its description of dimer spectra than any of the empirical effective potentials. It is also much better than earlier ab initio potentials and it is even somewhat better than the recent VRT(ASP-W) potential of Fellers et al. ${ }^{35}$ The latter is derived from the ASP-W potential of Millot and Stone. ${ }^{36}$ Fellers et al. fitted some of the parameters in this potential to a large number of transitions in the experimental VRT spectra. When supplemented with ab initio calculated three-body interactions, the SAPT-5st potential nicely reproduced $^{20}$ the experimental water trimer spectra as well. All of the results described in the present work are based on the SAPT5 st potential.

Previously, we computed the ground-state tunneling levels of $\left(\mathrm{H}_{2} \mathrm{O}\right)_{2}$ and $\left(\mathrm{D}_{2} \mathrm{O}\right)_{2}$ with energies up to $20 \mathrm{~cm}^{-1}$ above the ground state. The splittings of these energy levels are caused by the fact that the corresponding wave functions are linear combinations of functions localized in the eight equivalent minima of the dimer. Or, stated somewhat differently, they are caused by the tunneling of the system through the barriers separating the eight minima. We also calculated energy levels and wave functions in the region $50-200 \mathrm{~cm}^{-1}$ above the ground state. These yield intermolecular vibrational excitation energies, which we compared with experimental results. In assigning the levels, we provisionally used the names given to the modes by Saykally and co-workers. ${ }^{33,34}$ This nomenclature was first introduced in 1984 by Reimers and Watts. ${ }^{37}$ These workers considered all internal degrees of freedom and made a 12-dimensional normal-mode analysis of the dimer. From the experimental numbers obtained in Saykally's group, we know 
in retrospect that the harmonic frequencies calculated for the intermolecular normal modes (ranging from 115 to $782 \mathrm{~cm}^{-1}$ ) were in the correct energy range. Reimers and Watts did not present a detailed analysis of the nature of their modes but simply assumed that their modes were pure and were energy ordered as donor torsion (DT), acceptor wag (AW), acceptor twist (AT), stretch (S), in-plane-bend (IB), and out-of-plane bend (OB). The fact that this assignment is oversimplified is already clear from ref 37 , as an improvement of the normal-mode analysis by the approximate inclusion of anharmonicity gave a reordering of the levels. Incidentally, this anharmonicity correction raised the lower frequencies by about a factor of 3 and, thus, took the calculated levels far outside the correct energy range.

In this paper, we will first investigate the nature of the tunnelings and the intermolecular vibrations. To that end, we inspect the wave functions obtained in our earlier work. ${ }^{21}$ Because these wave functions depend on six coordinates, this inspection is highly nontrivial. Our general strategy is to plot cuts through the wave functions with two variables running and the other coordinates fixed at values corresponding to the global energy minimum of the dimer. The pair of running variables is chosen to describe, as closely as possible, the relevant path on the potential energy surface. First, we consider the different vibrational ground state wave functions, the energies of which are split by tunneling, along different tunneling paths that run from one equivalent minimum to another. Next, we consider librational/vibrational paths; the corresponding wave functions are vibrationally excited and have one or more nodal planes in addition to the tunneling nodes. By making a 6-dimensional intermolecular normal-mode analysis on the same SAPT-5st potential surface, we can compare the nature of the harmonic normal modes with the nature of the modes found from the inspection of the exact wave functions.

The second topic treated in this paper is the computation of dipole transition probabilities. Quantitative knowledge of the intensities of spectral lines is useful for several purposes. In the first place, it can guide spectroscopists in their search for new transitions. With not all of the VRT levels having been determined experimentally yet, we hope that some of the transitions that we predict to be (weakly) allowed will be observed in the future. In particular, we wish to point out that the $K=0$ acceptor tunneling splitting has not been measured separately. Only the sum of $K=0$ and $K=1$ splittings has been observed for $\left(\mathrm{H}_{2} \mathrm{O}\right)_{2}$, whereas for $\left(\mathrm{D}_{2} \mathrm{O}\right)_{2}$ the "experimental" value of the $K=0$ splitting is based on the assumption ${ }^{31}$ that the size of this splitting in the ground state is the same as in the excited state corresponding to the antisymmetric $\mathrm{O}-\mathrm{D}$ stretch vibration of the acceptor $\mathrm{D}_{2} \mathrm{O}$ monomer. Second, the line strengths of transitions that are forbidden in the harmonic approximation gauge the amount of anharmonicity of the potential. Last, but not least, we note that water dimers may play a role in atmospheric processes. As was recently discussed, ${ }^{38-43}$ dimers may be present in sufficient densities in the earth's atmosphere to give appreciable absorption of solar radiation. To model the solar absorption by dimers in the earth's atmosphere one must know the dipole transition probabilities.

\section{Theory and Computational Details}

The two water monomers have the reference geometry $X_{0}$ given in Table 1. The coordinates of the hydrogen bond acceptor $\mathrm{A}$ in the global minimum of the dimer SAPT-5st potential are obtained by left-multiplication of the matrix $X_{0}$ of column
TABLE 1: Reference Geometry $\left[a_{0}\right]$ of Water Monomer. O-H Distance: $1.8362 a_{0}$. Angle H-O-H: $104.69^{\circ}$

\begin{tabular}{rrrr}
\hline & $\mathrm{O}$ & \multicolumn{1}{c}{$\mathrm{H}_{1}$} & \multicolumn{1}{c}{$\mathrm{H}_{2}$} \\
\hline$x$ & 0.00000 & -1.45365 & 1.45365 \\
$y$ & 0.00000 & 0.00000 & 0.00000 \\
$z$ & 0.12463 & -0.99706 & -0.99706
\end{tabular}

TABLE 2: Geometry of Minimum Energy (SAPT-Fifth Potential) ${ }^{a}$

\begin{tabular}{lrrrrrr}
\hline & $\mathrm{O}_{1}$ & $\mathrm{H}_{1}$ & \multicolumn{1}{c}{$\mathrm{H}_{2}$} & \multicolumn{1}{c}{$\mathrm{O}_{2}$} & \multicolumn{1}{c}{$\mathrm{H}_{3}$} & \multicolumn{1}{c}{$\mathrm{H}_{4}$} \\
\hline$x$ & -0.10370 & 0.82960 & 0.82960 & 0.10954 & -0.18291 & -1.56971 \\
$y$ & 0.00000 & 1.45365 & -1.45365 & 0.00000 & 0.00000 & 0.00000 \\
$z$ & 5.46253 & 6.08472 & 6.08472 & -0.05945 & 1.75321 & -0.80201 \\
\multicolumn{5}{c}{ Unit: $a_{0}$. First three columns give the hydrogen bond acceptor A, } \\
the last three columns give the donor D, cf. Figure 1.
\end{tabular}
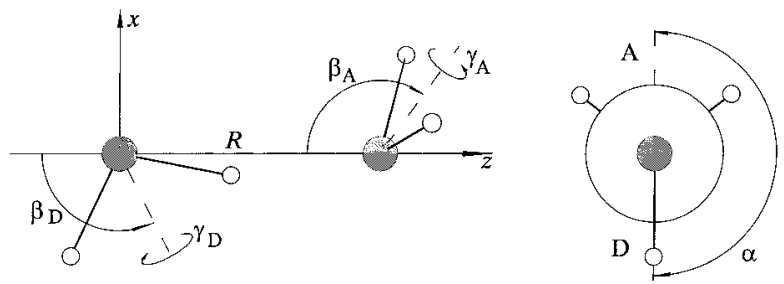

Figure 1. Euler angles describing the orientations of the two monomers in the water dimer. The figure on the right is a Newman projection in which we look along $\boldsymbol{R}$ from D to A.

vectors in this table with the matrix $\mathrm{R}_{A}=\mathrm{R}\left(180.0^{\circ}, 123.69^{\circ}\right.$, $90.0^{\circ}$ ), followed by a shift along the $z$-axis over $5.53166 a_{0}$. The latter number is the distance $R$ between the monomer centers of mass. The donor $\mathrm{D}$ is obtained from the reference geometry $\mathrm{X}_{0}$ by rotation with $\mathrm{R}_{\mathrm{D}}=\mathrm{R}\left(0.0^{\circ}, 118.49^{\circ}, 0.0^{\circ}\right)$. The rotation matrices

$$
\mathrm{R}\left(\alpha_{X}, \beta_{X}, \gamma_{X}\right) \equiv \mathrm{R}_{z}\left(\alpha_{X}\right) \mathrm{R}_{y}\left(\beta_{X}\right) \mathrm{R}_{z}\left(\gamma_{X}\right) \text { with } \mathrm{X}=\mathrm{A}, \mathrm{D}
$$

are in the active Euler convention, see for instance refs 44,45 for their explicit form. Thus, we obtain the equilibrium dimer geometry given in Table 2. See Figure 1 for a graphical illustration of the Euler angles and the equilibrium geometry of the dimer. Note that it has reflection symmetry with respect to the $x z$-plane, point group $C_{s}$.

As we already discussed in the Introduction, several kinds of motion of a dimer consisting of rigid monomers can be distinguished. In the first place, the dimer may tunnel between eight equivalent-permutationally distinct-equilibrium structures. Three different tunneling processes allow the dimer to interconvert between the minima: acceptor tunneling, donoracceptor interchange, and bifurcation tunneling. The first tunneling is an interchange of the acceptor protons, whereas donor-acceptor interchange and bifurcation tunneling both involve breaking of the hydrogen bond. These tunnelings are illustrated in Figure 2, the level splitting pattern caused by these tunneling processes is shown in Figure 3. The second kind of dimer motion is the intermolecular vibration, where we distinguish six fundamental modes following ref 37 . Finally, the dimer rotates in isotropic space. With respect to rotation the dimer behaves as a prolate near-symmetric rotor. The rotational quantum number $K \equiv K_{a}$ is the projection of the total angular momentum $\boldsymbol{J}$ with quantum number $J$ on the long axis $a$.

To describe the symmetry of the dimer, we label the acceptor protons by 1 and 2 and the donor protons by 3 and 4 . These four protons are identical, as are the two oxygens $\mathrm{O}_{1}$ and $\mathrm{O}_{2}$. Not all $4 ! \times 2$ ! permutations are feasible. The permutation- 


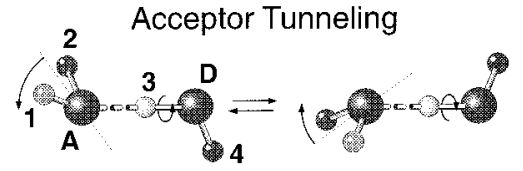

Donor-Acceptor Interchange Tunneling

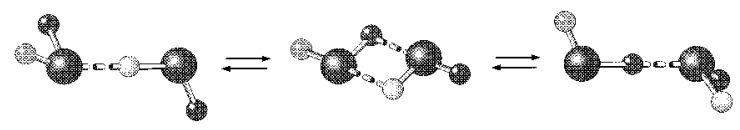

Donor (Bifurcation) Tunneling

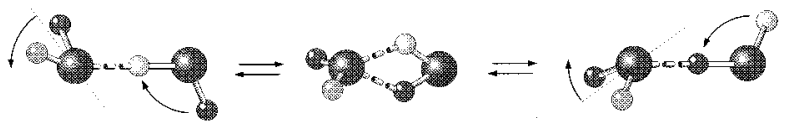

Figure 2. Three different hydrogen bond rearrangement processes in the water dimer which connect the eight equivalent, permutationally distinct, equilibrium structures: acceptor tunneling with PI operation (12), donor-acceptor interchange tunneling with PI operation $\left(\mathrm{O}_{1} \mathrm{O}_{2}\right)$ (1423), and bifurcation (or donor) tunneling with PI operation (12)(34).
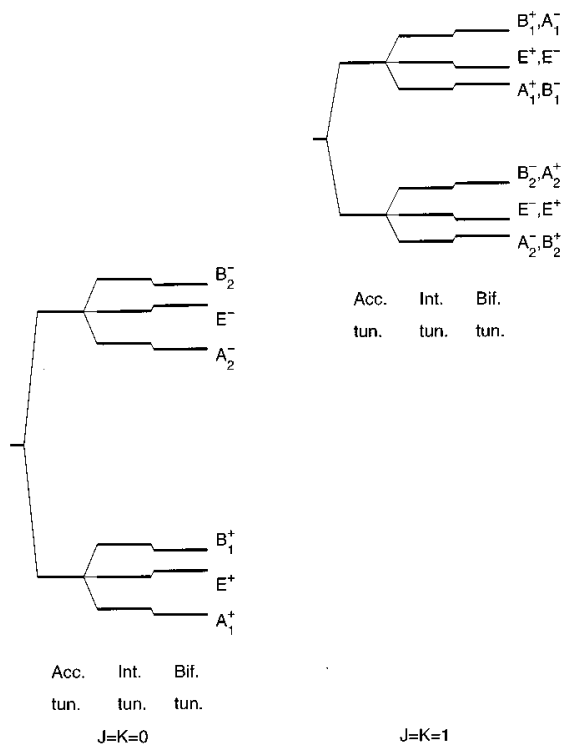

Figure 3. Tunneling splitting pattern of the rovibrational levels of the water dimer by the mechanisms shown in Figure 2.

inversion (PI) group, which by definition consists of feasible permutations only, is generated by (12), (34), $P_{\mathrm{AB}} \equiv\left(\mathrm{O}_{1} \mathrm{O}_{2}\right)$ (13)(24) and $E^{*}$. The latter operation is the inversion with respect to the dimer center of mass. This PI group is denoted by $G_{16}$. Note, parenthetically, that reflection in the mirror plane of the equilibrium structure is equivalent to $(12)^{*} \equiv(12) E^{*}$. The VRT levels of the water dimer are labeled by the irreducible representations (irreps) of $G_{16}: A_{1,2}^{ \pm}, B_{1,2}^{ \pm}$, and $E^{ \pm}$. The acceptor tunneling motion yields a relatively large splitting between the $A_{1}^{ \pm}, E^{ \pm}, B_{1,2}^{ \pm}$levels on one hand, and the $A_{2}^{\mp}, E^{\mp}, B_{2}^{\mp}$ levels on the other, whereas interchange tunneling yields a smaller splitting between the respective $A$ and $B$ levels $\left(A_{1}^{+}\right.$and $B_{1}^{+}$ etc.), cf. Figure 3 . The bifurcation (donor) tunneling causes no splitting, but leads to a shift of the $E$ symmetry levels and an opposite shift of the levels of $A$ and $B$ symmetry.

For the convenience of the reader we summarize briefly how we obtained the VRT functions. ${ }^{21}$ These functions are eigenfunctions of the Hamiltonian

$$
\begin{aligned}
H=T_{A}+T_{B}+ & \frac{1}{2 \mu_{A B} R^{2}}\left[-\hbar^{2} R \frac{\partial^{2}}{\partial R^{2}} R+J^{2}+j_{A B}^{2}-\right. \\
& \left.2 j_{\mathrm{AB}} \cdot J\right]+V\left(R, \beta_{A}, \gamma_{A}, \beta_{B}, \gamma_{B}, \alpha_{\mathrm{A}}-\alpha_{B}\right)
\end{aligned}
$$

where $\omega_{X} \equiv\left(\alpha_{X}, \beta_{X}, \gamma_{X}\right)(X \in\{A, B\})$ are the Euler angles of the respective monomers. These Euler angles, the same as those used in eq (1), are defined with respect to the dimer two-angle embedded frame ${ }^{46}$ obtained by rotating a space-fixed frame over the polar angles $(\Phi, \Theta)$ of the intermolecular vector $\boldsymbol{R} \equiv \boldsymbol{R}_{A B}$ (of length $R$ ) connecting the centers of mass of monomers $\mathrm{A}$ and B such that the $z$ axis of the rotated system is along $\boldsymbol{R}$. The operator $\boldsymbol{J}$ is the total angular momentum, $\boldsymbol{j}_{A B}=\boldsymbol{j}_{A}+\boldsymbol{j}_{B}$ is the sum of the monomer angular momenta, and $\mu_{A B}$ is the dimer reduced mass. The kinetic energy operator of monomer $\mathrm{X}$ is given by

$$
T_{X}=A_{X}\left(j_{X x}^{\mathrm{BF}}\right)^{2}+B_{X}\left(j_{X y}^{\mathrm{BF}}\right)^{2}+C_{X}\left(j_{X z}^{\mathrm{BF}}\right)^{2}
$$

with the rotational constants $A_{\mathrm{X}}, B_{\mathrm{X}}$ and $C_{\mathrm{X}}$. The superscript $\mathrm{BF}$ (body-fixed) implies here that $x, y$, and $z$ refer to the components of $\boldsymbol{j}_{X}$ along the principal axes of monomer X. ${ }^{46}$ The quantity $V$ is the SAPT-5st potential. ${ }^{21}$

We determine the bound states of $H$ variationally and expand the wave functions in the basis

$$
\begin{array}{r}
|n, \boldsymbol{I} ; J M\rangle=\Phi_{n}(R)\left[\frac{\left(2 j_{A}+1\right)\left(2 j_{B}+1\right)(2 J+1)}{256 \pi^{5}}\right]^{1 / 2} \times \\
\sum_{m_{A}=-j_{A}}^{j_{A}} \sum_{m_{B}=-j_{B}}^{j_{B}} D_{m_{A} k_{A}}^{\left(j_{A}\right)}\left(\omega_{A}\right)^{*} D_{m_{B} k_{B}}^{\left(j_{B}\right)}\left(\omega_{B}\right)^{*}\left\langle j_{A} m_{A} ; j_{B} m_{\mathrm{B}} \mid j_{A B} K\right\rangle \times \\
D_{M K}^{(J)}(\Phi, \Theta, 0)^{*}
\end{array}
$$

in which $\boldsymbol{I} \equiv\left\{j_{A}, k_{A}, j_{B}, k_{B}, j_{A B}, K\right\},\left\langle j_{A} m_{A} ; j_{B} m_{B} \mid j_{A B} K\right\rangle$ is a Clebsch-Gordan coupling coefficient, and $D_{m_{X} k_{X}}^{\left(j_{X}\right)}\left(\omega_{X}\right)$ is a Wigner $D$-function. The basis of eq (4) extends to functions with $j_{A}=j_{B}=11$ inclusive. For the radial basis $\Phi_{n}(R)$, we use a contracted sinc function discrete variable representation (DVR). ${ }^{47,48}$ The basis is adapted to the symmetry group $G_{16}$ and the eigenvectors of the Hamiltonian are computed separately for each irrep. The small, off-diagonal Coriolis coupling is neglected, which makes $K$ an exact quantum number. ${ }^{21}$

The components of the dipole moment operator $\mu_{k}^{\mathrm{BF}}\left(R, \omega_{A}\right.$, $\left.\omega_{B}\right)$ with respect to the body-fixed dimer frame are expanded in the $\operatorname{basis}^{46}(L \equiv 1)$

$$
\begin{aligned}
B_{\Lambda, k}^{\mathrm{BF}}\left(\omega_{A}, \omega_{B}\right)=\sum_{M_{A}, M_{B}} D_{M_{A} K_{A}}^{\left(L_{A}\right)}\left(\omega_{A}\right)^{*} D_{M_{B} K_{B}}^{\left(L_{B}\right)}\left(\omega_{B}\right)^{*} & \\
& \left\langle L_{A} M_{A} ; L_{B} M_{B} \mid L k\right\rangle
\end{aligned}
$$

where $\left\langle L_{A} M_{A} ; L_{B} M_{B} \mid L k\right\rangle$ is a Clebsch-Gordan coefficient and $\Lambda=\left\{L_{A}, L_{B}, L, K_{A}, K_{B}\right\}$. The dipole then becomes

$$
\mu_{k}^{\mathrm{BF}}\left(R, \omega_{A}, \omega_{B}\right)=\sum_{\Lambda} d_{\Lambda, k}^{\mathrm{BF}}(R) B_{\Lambda, k}^{\mathrm{BF}}\left(\omega_{A}, \omega_{B}\right)
$$

where $k=0$ refers to the component parallel to the dimer $z$-axis (the vector $\boldsymbol{R}$ ) and $k= \pm 1$ to the perpendicular components. The relation between the SF and BF dipole components is

$$
\mu_{m}^{\mathrm{SF}}=\sum_{k} \mu_{k}^{\mathrm{BF}}\left(R, \omega_{A}, \omega_{B}\right) D_{m k}^{(1)}(\Phi, \Theta, 0)^{*}
$$


The matrix elements of the dipole moment operator $\boldsymbol{\mu}^{\mathrm{SF}}$ are ${ }^{49}$

$$
\begin{gathered}
\left\langle n^{\prime}, \boldsymbol{I}^{\prime} ; J^{\prime} M^{\prime}\left|\mu_{m}^{\mathrm{SF}}\right| n, \boldsymbol{I} ; J M\right\rangle=(-1)^{M^{\prime}+j_{A}^{\prime}+j_{B}{ }_{B}+j_{A B}^{\prime}+k_{A}^{\prime}+k_{B}^{\prime}} \times \\
{\left[\left(2 J^{\prime}+1\right)(2 J+1)\left(2 j_{A}^{\prime}+1\right)\left(2 j_{B}^{\prime}+1\right)\left(2 j_{\mathrm{A}}+1\right)\left(2 j_{\mathrm{B}}+1\right)\right.} \\
\left.\left(2 j_{A B}^{\prime}+1\right)\left(2 j_{A B}+1\right)\right]^{1 / 2}\left(\begin{array}{lll}
J^{\prime} & 1 & J \\
-M^{\prime} & m & M
\end{array}\right) \sum_{k}\left(\begin{array}{lll}
J^{\prime} & 1 & J \\
-K^{\prime} & k & K
\end{array}\right) \sum_{\Lambda} \\
(2 L+1)^{1 / 2}\left\langle\Phi_{n^{\prime}}(R)\left|d_{\Lambda, k}^{\mathrm{BF}}(R)\right| \Phi_{\mathrm{n}}(R)\right\rangle\left(\begin{array}{lll}
j_{A}^{\prime} & L_{A} & j_{A} \\
-k_{A}^{\prime} & K_{A} & k_{A}
\end{array}\right) \\
\left(\begin{array}{llll}
j_{B}^{\prime} & L_{B} & j_{B} \\
-k_{B}^{\prime} & K_{B} & k_{B}
\end{array}\right)\left(\begin{array}{lll}
j_{A B}^{\prime} & L & j_{A B} \\
-K^{\prime} & k & K
\end{array}\right)\left\{\begin{array}{llll}
j_{A}^{\prime} & L_{A} & j_{A} \\
j_{B}^{\prime} & L_{B} & j_{B} \\
j_{A B}^{\prime} & L & j_{A B}
\end{array}\right\}
\end{gathered}
$$

where the expressions in round and curly brackets are $3 j$ and $9 j$ symbols, respectively. If we assume that the transition moments are purely determined by the monomers' dipole moments, the operator $d_{\Lambda, k}^{\mathrm{BF}}$ becomes simply

$$
d_{\Lambda, k}^{\mathrm{BF}}=\delta_{L_{B} 0} \delta_{K_{B} 0} \delta_{L_{A} 1} \delta_{L_{A} L} \mu_{K_{A}}+\delta_{L_{A} 0} \delta_{K_{A} 0} \delta_{L_{B} 1} \delta_{L_{B} L} \mu_{K_{B}}
$$

independent of $k$, where $\mu_{K_{A}} \equiv Q_{K_{A}}^{1}$ and $\mu_{K_{B}} \equiv Q_{K_{B}}^{1}$ are the components of the permanent dipoles on the monomers, expressed in the monomer frames. For the dipole moment induced on monomer A by the permanent multipole moments $Q_{K_{B}}^{L_{B}}$ of monomer B, the coefficients in eq (6) are

$$
\begin{array}{r}
d_{\Lambda, k}^{\mathrm{BF}}(A \leftarrow B)= \\
\sum_{l_{A}}(-1)^{k+l_{\mathrm{A}}}\left[\left(2 L_{\mathrm{A}}+1\right)\left(2 l_{\mathrm{A}}+2 L_{\mathrm{B}}+1\right)\right. \\
(2 L+1)]^{1 / 2}\left(\begin{array}{lll}
2 l_{\mathrm{A}}+2 L_{B} \\
2 l_{A}
\end{array}\right)\left(\begin{array}{lll}
l_{\mathrm{A}}+L_{B} & L & 1 \\
0 & k & -k
\end{array}\right) \times \\
\left\{\begin{array}{lll}
l_{\mathrm{A}}+L_{B} & L_{B} & l_{A} \\
L_{A} & 1 & L
\end{array}\right\} Q_{K_{B}}^{L_{B}} \alpha_{K_{A}}{ }^{\left(1, l_{A} ; L_{A}\right)} R^{-l_{A}-L_{B}-1}
\end{array}
$$

where $\alpha_{K_{A}}{ }^{\left(1, l_{A} ; L_{A}\right)}$ are the irreducible components of the (mixed)

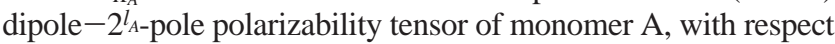
to the monomer frame. ${ }^{46,49}$ The expression in curly brackets is a $6 j$ symbol. The expansion coefficients $d_{\Lambda, k}^{\mathrm{BF}}(B \leftarrow A)$ of the dipole moment induced on monomer $B$ by the permanent multipole moments of monomer A can be written similarly. The transition moments given in section 4 are calculated using dipole moments and polarizabilities only $\left(l_{A}=l_{B}=1\right)$. The monomer dipole moment $Q_{0}^{1} \equiv \mu_{z}=-0.7277 e a_{0}$ and dipole polarizability $\alpha_{x x}=9.988 \alpha_{0}^{3}, \alpha_{y y}=8.910 \alpha_{0}^{3}, \alpha_{z z}=9.470 a_{0}^{3}$, with the atomic coordinates defined as in Table 1, were taken from ref 50. Induction by quadrupole and higher multipole moments, dispersion, and short-range (penetration and exchange) contributions to the dipole moment function were neglected, as we expect these to be considerably smaller than the contributions that we do include, and the transition intensities cannot be measured very accurately anyway.

We end this theory section by briefly explaining how we computed the harmonic frequencies and normal modes from the same SAPT-5st potential as the nearly exact VRT wave functions. We used the standard Wilson GF-matrix method ${ }^{51,52}$ to solve the six-dimensional harmonic oscillator problem. The $6 \times 6$ force constant matrix $\boldsymbol{F}$ was obtained from the potential by numerical differentiation with respect to the distance $R$ and the angles $\beta_{A}, \gamma_{A}, \beta_{B}, \gamma_{B}, \alpha \equiv \alpha_{A}-\alpha_{B}$. The inverse metric tensor $\boldsymbol{G}$ corresponding to these coordinates was derived by starting from 12 dimer coordinates, six for each monomer. The latter six are the three Cartesian coordinates of the monomer center of mass and the three Euler angles describing its orientation, both with respect to a space-fixed frame. For the Euler angles, we took the expression for the metric tensor that can be extracted from the well-known kinetic energy operator. ${ }^{44}$ To compute the $6 \times 6$ matrix $\boldsymbol{G}$ we used the Jacobian of the transformation from those 12 monomer coordinates to the six dimer coordinates, defined with respect to a body-fixed dimer frame.

\section{Tunneling and Vibrations in the Water Dimer}

3.1. Method of Analysis. From here on, we will often refer to the monomers as D (the hydrogen bond donor) and A (the acceptor) rather than $\mathrm{A}$ and $\mathrm{B}$. The nomenclature of the normal modes by Reimers and Watts ${ }^{37}$ suggests that a good approximation to these modes is given by infinitesimal changes in the monomer Euler angles and in the distance $R$ from their respective equilibrium values (cf. Figure 1 ). Thus, one associates donor torsion (DT) with changes in $\alpha \equiv \alpha_{D}-\alpha_{A}$, acceptor wag (AW) with $\beta_{A}$, in-plane bend (IB) with $\beta_{D}$, stretch (S) with $R$, acceptor twist (AT) with $\gamma_{A}$, and out-of-plane bend (OB) with $\gamma_{D}$. However, a priori it is not evident that each of the normal modes is described by the infinitesimal variation in one Euler angle only. And, indeed, as we will see below, the harmonic modes are mixtures of different linearized coordinates. Moreover, we expect the harmonic approximation not to be valid for the water dimer as the intermolecular potential is not at all quadratic in the Euler angles. Further, we recall that a wellknown disadvantage of the harmonic approximation is that it departs from a single well-defined equilibrium geometry so that it cannot describe tunneling processes.

In this section, we will study intermolecular vibrations and tunneling effects. To that end, we analyze the exact nondegenerate VRT wave functions. The nodal planes in the vibrationally excited states $(J, K, v)$ are orthogonal to the vibrational directions and, hence, allow a definite characterization of the modes. The quantum number $v$ labels the vibrational states in increasing energy; $v=0$ gives the vibrational ground state. The states are further characterized by their reflection ( $C_{s}$ point group) symmetry: $A^{\prime}$ or $A^{\prime \prime}$. The wave functions corresponding to the upper tunneling levels have nodal surfaces that are orthogonal to the tunneling paths.

By plotting the wave functions as functions of the above coordinates, the classification of the modes may be determined. In the plots, two coordinates are taken as variables at a time, the others kept fixed at suitable values. If not mentioned otherwise, we choose the equilibrium values for the fixed coordinates. For the distance $R$, we choose the point on the DVR grid (step size $\approx 0.1 a_{0}$ ) nearest to the equilibrium distance; there the amplitudes of the nonstretching modes are close to their maxima. The amount of stretch variation may be described quantitatively by $\Delta R \equiv\left\langle(R-\langle R\rangle)^{2}\right\rangle^{1 / 2}$ as well as by the expectation value $\langle R\rangle$. Both are a measure of the displacement. Tables 3 and 4 contain the values for all the vibrational states considered here. The vibrations resembling hindered internal rotations (librations) can be characterized quantitatively by the extrema of the wave functions. These are listed in Tables 5 and 6 for the $\mathrm{H}_{2} \mathrm{O}$ and $\mathrm{D}_{2} \mathrm{O}$ dimer, respectively. The paths from the minima to the maxima correspond to the directions of the vibrations.

The states with $|K|=1$ are more complicated than those with $K=0$ because the internal and external rotations around the long axis do not separate. After substitution of the Wigner $D$ 
TABLE 3: Energies, Radial Expectation Values and Displacements of $\left(\mathrm{H}_{2} \mathrm{O}\right)_{2}$ States $(J, K, v)^{a}$

\begin{tabular}{rccrlcc}
\hline & state & $C_{s}$ irrep & energy & mode & $\langle R\rangle$ & $\Delta R$ \\
\hline$J=K=0$ & $(0,0,0)$ & $A^{\prime}$ & 0.00 & GS & 5.6326 & 0.2201 \\
& $(0,0,1)$ & $A^{\prime \prime}$ & 85.72 & DT & 5.6369 & 0.2233 \\
& $(0,0,2)$ & $A^{\prime}$ & 111.98 & AW & 5.6482 & 0.2514 \\
& $(0,0,3)$ & $A^{\prime \prime}$ & 121.11 & AT & 5.6342 & 0.2252 \\
& $(0,0,4)$ & $A^{\prime}$ & 139.83 & (DT $)^{2}$ & 5.6185 & 0.2330 \\
& $(0,0,5)$ & $A^{\prime}$ & 165.64 & S & 5.7177 & 0.3403 \\
$J=|K|=1$ & $(0,0,6)$ & $A^{\prime \prime}$ & 185.18 & DT + AW & 5.6918 & 0.3190 \\
& $(1,1,0)$ & $A^{\prime}$ & 7.37 & GS & 5.6308 & 0.2195 \\
& $(1,1,1)$ & $A^{\prime \prime}$ & 85.42 & DT & 5.6338 & 0.2225 \\
& $(1,1,2)$ & $A^{\prime}$ & 119.22 & AW & 5.6319 & 0.2317 \\
& $(1,1,3)$ & $A^{\prime}$ & 136.85 & (DT $)^{2 b}$ & 5.6477 & 0.2559 \\
& $(1,1,4)$ & $A^{\prime}$ & 154.50 & S & 5.7135 & 0.3345 \\
& $(1,1,5)$ & $A^{\prime \prime}$ & 170.10 & AT & 5.6064 & 0.2247
\end{tabular}

${ }^{a}$ The energy (in $\mathrm{cm}^{-1}$ ) of each vibrational state, relative to the ground state (GS), is the average over all tunneling components belonging to the one-dimensional $A$ and $B$ irreps, see ref 21. For $J=K=0$ the results $\langle R\rangle$ and $\Delta R$ refer to the $A_{1}^{+}$and $A_{2}^{+}$components, for $J=|K|=$ 1 to the $B_{1}^{-}$components. ${ }^{b}$ The assignment of this mode from its nodal character is uncertain, because of the intertwinement of the nodal planes in the torsional coordinate $\alpha$ and in the angle $\bar{\alpha}$ in the overall rotation wave function with $K= \pm 1$.

TABLE 4: Energies, Radial Expectation Values and Displacements of $\left(\mathrm{D}_{2} \mathrm{O}\right)_{2}$ States. for Explanations, See Table 3

\begin{tabular}{lccrlcc}
\hline & state & $C_{s}$ irrep & energy & mode & $\langle R\rangle$ & $\Delta R$ \\
\hline$J=K=0$ & $(0,0,0)$ & $A^{\prime}$ & 0.00 & GS & 5.6309 & 0.2078 \\
& $(0,0,1)$ & $A^{\prime \prime}$ & 67.35 & DT & 5.6430 & 0.2138 \\
& $(0,0,2)$ & $A^{\prime}$ & 91.58 & AW & 5.6476 & 0.2276 \\
& $(0,0,3)$ & $A^{\prime \prime}$ & 92.04 & AT & 5.6283 & 0.2090 \\
& $(0,0,4)$ & $A^{\prime}$ & 123.07 & (DT) & 5.6379 & 0.2151 \\
& $(0,0,5)$ & $A^{\prime}$ & 146.82 & S & 5.6470 & 0.2493 \\
$J=|K|=1$ & $(0,0,6)$ & $A^{\prime \prime}$ & 146.06 & DT + AW & 5.6448 & 0.2253 \\
& $(1,1,0)$ & $A^{\prime}$ & 3.96 & GS & 5.6301 & 0.2075 \\
& $(1,1,1)$ & $A^{\prime \prime}$ & 69.68 & DT & 5.6413 & 0.2132 \\
& $(1,1,2)$ & $A^{\prime}$ & 93.18 & AW & 5.6329 & 0.2159 \\
& $(1,1,3)$ & $A^{\prime}$ & 100.79 & (DT) ${ }^{2 a}$ & 5.6368 & 0.2217 \\
& $(1,1,4)$ & $A^{\prime \prime}$ & 122.61 & DT + AW & 5.6383 & 0.2154 \\
& $(1,1,5)$ & $A^{\prime}$ & 146.15 & S & 5.6621 & 0.2705
\end{tabular}

${ }^{a}$ The assignment of this mode from its nodal character is uncertain, because of the intertwinement of the nodal planes in the torsional coordinate $\alpha$ and in the angle $\bar{\alpha}$ in the overall rotation wave function with $K= \pm 1$.

functions in the basis of eq (4), $D_{m_{X} k_{X}}^{\left(j_{X}\right)}\left(\omega_{X}\right)^{*}=\exp \left(i m_{X} \alpha_{X}\right)$ $d_{m_{X} k_{X}}^{j_{X}}\left(\beta_{X}\right) \exp \left(i k_{X} \gamma_{X}\right)$, the function $\exp \left(i m_{D} \alpha_{D}\right) \exp \left(i m_{A} \alpha_{A}\right)$ can be rewritten in terms of the internal angle $\alpha \equiv \alpha_{D}-\alpha_{A}$ and the overall rotation angle $\bar{\alpha} \equiv\left(\alpha_{D}+\alpha_{A}\right) / 2$ as $\exp \left[i\left(m_{D}-m_{A}\right) \alpha /\right.$ 2] $\exp (\mathrm{i} K \bar{\alpha})$, with $K=m_{D}+m_{\mathrm{A}}$. Each basis function in eq (4) can then be factorized into an external part: $\exp (i K \bar{\alpha}) D_{M K}^{(J)}(\Phi$, $\Theta, 0)^{*}=D_{M K}^{(J)}(\Phi, \Theta, \bar{\alpha})^{*}$ depending on the overall rotation angles $\Theta, \Phi, \bar{\alpha}$ and an internal part depending on $\beta_{D}, \gamma_{D}, \beta_{A}$, $\gamma_{A}$, and $\alpha$ [cf. eq (B19) in ref 46]. However, the adaptation of the basis to the $G_{16}$ symmetry gives linear combinations of such basis functions, see Table 2 of ref 21 , and the exact wave functions are linear combinations as well. Such combinations are no longer separable in $\alpha$ and $\bar{\alpha}$ and it is not trivial to view the internal part of the wave functions, without being hindered by the nodal behavior of the external part. We solved this problem by first plotting the wave function as a function of both $\alpha_{D}$ and $\alpha_{A}$ and then fixing the external rotation angle $\bar{\alpha}=$ $\left(\alpha_{D}+\alpha_{A}\right) / 2$ at a suitable value, while choosing the path of torsional rotation, such that one avoids the nodes in the external wave function. To get pure real or imaginary vibrational functions as in the case of $K=0$, the angles $\Phi$ and $\Theta$ as well as the quantum number $M$ must be chosen adequately.
TABLE 5: Locations of Maxima and Minima in the $\left(\mathrm{H}_{2} \mathrm{O}\right)_{2}$ Wave Functions at $\boldsymbol{R}=\mathbf{5 . 5 6} a_{0}\left(R_{e}=\mathbf{5 . 5 3} a_{0}\right)$

\begin{tabular}{cccccccr}
\hline & state & irrep & $\alpha$ & $\beta_{D}$ & \multicolumn{1}{c}{$\gamma_{D}$} & \multicolumn{1}{c}{$\beta_{A}$} & \multicolumn{1}{c}{$\gamma_{A}$} \\
\hline & equil geom & & 180.00 & 118.49 & 0.00 & 123.69 & 90.00 \\
\hline$J=K=0$ & $(0,0,0)\left(A^{\prime}\right)$ & $A_{1}^{+}$ & 180.00 & 119.96 & 0.00 & 134.93 & 90.00 \\
& $(0,0,1)\left(A^{\prime \prime}\right)$ & $A_{2}^{+}$ & 146.10 & 116.04 & 1.93 & 150.16 & 124.13 \\
& & & 213.90 & & -1.93 & & 55.87 \\
& $(0,0,2)\left(A^{\prime}\right)$ & $B_{2}^{-}$ & 180.00 & 108.15 & 0.00 & 108.03 & 90.00 \\
& & & & 128.95 & & 180.00 & \\
& $(0,0,3)\left(A^{\prime \prime}\right)$ & $A_{2}^{+}$ & 159.97 & 119.60 & -15.37 & 130.40 & 62.20 \\
& & & 200.03 & & 15.37 & & 117.80 \\
& $(0,0,4)\left(A^{\prime}\right)$ & $A_{1}^{+}$ & 180.00 & 123.94 & 0.00 & 134.67 & 90.00 \\
& & & 115.07 & & -4.91 & & 120.73 \\
& & & 244.93 & & 4.91 & & 59.27 \\
& $(0,0,5)\left(A^{\prime}\right)$ & $A_{1}^{+}$ & 180.00 & 124.99 & 0.00 & 152.02 & 90.00 \\
& $(0,0,6)\left(A^{\prime \prime}\right)$ & $B_{1}^{-}$ & 104.12 & 120.70 & -8.71 & 159.59 & 144.39 \\
& & & 255.88 & & 8.71 & & 35.61 \\
$J=|K|=1$ & $(1,1,0)\left(A^{\prime}\right)$ & $B_{1}^{-}$ & 180.00 & 119.77 & 0.00 & 133.98 & 90.00 \\
& $(1,1,1)\left(A^{\prime \prime}\right)$ & $B_{1}^{-}$ & 147.20 & 116.51 & 1.63 & 138.83 & 109.61 \\
& & & 212.80 & & -1.63 & & 70.39 \\
& $(1,1,2)\left(A^{\prime}\right)$ & $B_{1}^{-}$ & 180.00 & 107.36 & 0.00 & 107.51 & 90.00 \\
& & & & 129.08 & & 180.00 & \\
& $(1,1,3)\left(A^{\prime}\right)$ & $B_{1}^{-}$ & 180.00 & 108.62 & 0.00 & 108.98 & 90.00 \\
& $(1,1,4)\left(A^{\prime}\right)$ & $B_{1}^{-}$ & 180.00 & 125.46 & 0.00 & 150.00 & \\
& $(1,1,5)\left(A^{\prime \prime}\right)$ & $B_{1}^{-}$ & 171.06 & 112.17 & 10.81 & 130.62 & 124.66 \\
& & & 188.94 & & -10.81 & & 55.34
\end{tabular}

TABLE 6: Locations of Maxima and Minima in the $\left(D_{2} \mathrm{O}\right)_{2}$ Wave Functions at $R=5.57 a_{0}\left(R_{e}=5.54 a_{0}\right)$

\begin{tabular}{|c|c|c|c|c|c|c|c|}
\hline & state & irrep & $\alpha$ & $\beta_{D}$ & $\gamma_{D}$ & $\beta_{A}$ & $\gamma_{A}$ \\
\hline & equil geom & & 180.00 & 116.72 & 0.00 & 125.46 & 90.00 \\
\hline \multirow[t]{14}{*}{$J=K=0$} & $(0,0,0)\left(A^{\prime}\right)$ & $A_{1}^{+}$ & 180.00 & 117.90 & 0.00 & 132.48 & 90.00 \\
\hline & $(0,0,1)\left(A^{\prime \prime}\right)$ & $A_{2}^{+}$ & 148.86 & 115.54 & 1.67 & 137.61 & 107.06 \\
\hline & & & 211.14 & & -1.67 & & 72.94 \\
\hline & $(0,0,2)\left(A^{\prime}\right)$ & $B_{2}^{-}$ & 180.00 & 107.39 & 0.00 & 110.58 & 90.00 \\
\hline & & & & 127.79 & & 164.58 & \\
\hline & $(0,0,3)\left(A^{\prime \prime}\right)$ & $A_{2}^{+}$ & 163.80 & 117.80 & -13.20 & 129.36 & 67.68 \\
\hline & & & 196.20 & & 13.20 & & 112.32 \\
\hline & $(0,0,4)\left(A^{\prime}\right)$ & $A_{1}^{+}$ & 180.00 & 126.19 & 0.00 & 148.32 & 90.00 \\
\hline & & & 120.92 & & -2.06 & & 113.86 \\
\hline & & & 239.08 & & 2.06 & & 66.14 \\
\hline & $(0,0,5)\left(A^{\prime}\right)$ & $A_{1}^{+}$ & 180.00 & 107.01 & 0.00 & 106.42 & 90.00 \\
\hline & & & & 126.58 & & 160.76 & \\
\hline & $(0,0,6)\left(A^{\prime \prime}\right)$ & $B_{1}^{-}$ & 142.82 & 121.55 & -7.63 & 166.86 & 9.01 \\
\hline & & & 217.18 & & 7.63 & & 170.99 \\
\hline \multirow[t]{9}{*}{$J=|K|=1$} & $(1,1,0)\left(A^{\prime}\right)$ & $B_{1}^{-}$ & 180.00 & 117.86 & 0.00 & 132.29 & 90.00 \\
\hline & $(1,1,1)\left(A^{\prime \prime}\right)$ & $B_{1}^{-}$ & 150.06 & 115.59 & 0.98 & 135.20 & 104.55 \\
\hline & & & 209.94 & & -0.98 & & 75.45 \\
\hline & $(1,1,2)\left(A^{\prime}\right)$ & $B_{1}^{-}$ & 180.00 & 107.47 & 0.00 & 110.97 & 90.00 \\
\hline & & & & 127.92 & & & \\
\hline & $(1,1,3)\left(A^{\prime}\right)$ & $B_{1}^{-}$ & 180.00 & 107.62 & 0.00 & 110.70 & 90.00 \\
\hline & & & & 127.70 & & 164.85 & \\
\hline & $(1,1,4)(A$ & $B_{1}^{-}$ & 149.78 & & 0.90 & & 105.10 \\
\hline & $(1,1,5)\left(A^{\prime}\right)$ & $B_{1}^{-}$ & $\begin{array}{l}210.22 \\
180.00\end{array}$ & 125.69 & $\begin{array}{r}-0.90 \\
0.00\end{array}$ & 177.61 & $\begin{array}{l}74.90 \\
90.00\end{array}$ \\
\hline
\end{tabular}

The three different tunneling paths in the water dimer, see Figure 2, have been investigated by Wales. ${ }^{53}$ Wales defines a tunneling path as a one-dimensional (curved) route of minimum energy over the six-dimensional intermolecular potential surface from one global minimum to another, equivalent, minimum. In reality, however, tunneling does not take place on a onedimensional path because the zero-point kinetic energy and the strong coupling in the potential between all of the six intermolecular degrees of freedom lead to a delocalization of the wave function in all directions. Still, we expect to obtain useful insight into the nature of the three different tunneling processes if we 

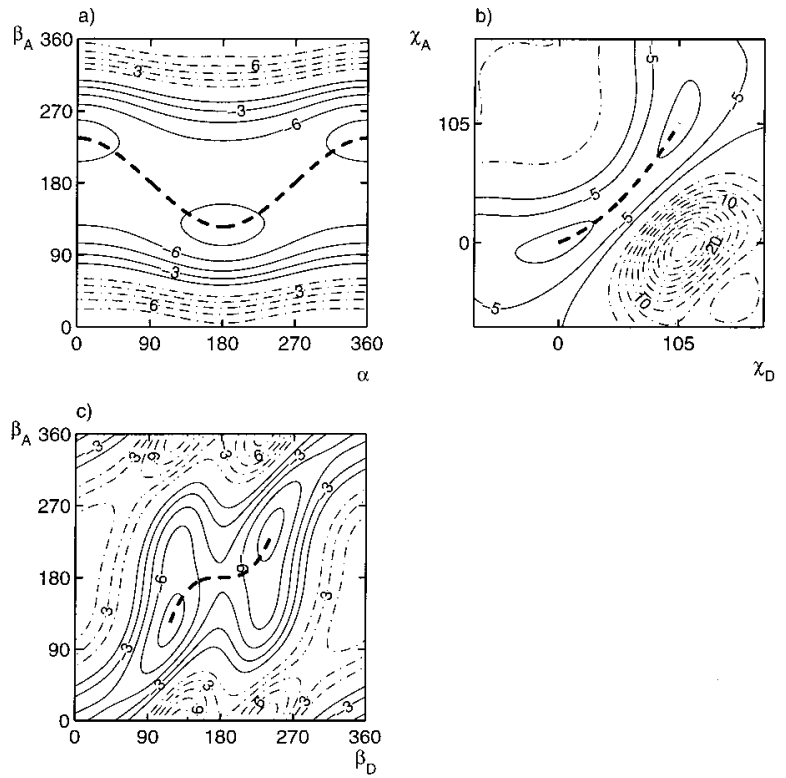

Figure 4. Cuts through the potential surface (in $\mathrm{m} E_{h}$ ) relevant for the different tunneling processes in the $\mathrm{H}_{2} \mathrm{O}$ dimer. Closed contours denote negative energy, dot-dashed contours positive energy. The tunneling paths are indicated by a bold dashed line. (a) Acceptor tunneling; (b) Interchange tunneling; (c) Bifurcation tunneling. The angles $\chi_{D}$ and $\chi_{A}$ are defined in section 3.1 .

plot two-dimensional cuts through the wave functions in coordinate planes that contain the one-dimensional paths found by Wales. Figure 4 shows the three corresponding twodimensional cuts through the six-dimensional intermolecular potential surface.

The acceptor tunneling path, according to Wales, may be described as a rotation of the acceptor around the $y$ axis from $\beta_{A}$ to $360^{\circ}-\beta_{A}$ (Figure $4 \mathrm{a}$ ), with a simultaneous torsion $\alpha$ over $180^{\circ}$, see Figure 2 .

The preferred acceptor-donor interchange tunneling path is a geared rotation of the acceptor and donor. ${ }^{53}$ At the end-point of this concerted rotation the acceptor and donor have changed roles. It is not trivial to find a two-dimensional surface in the six-dimensional coordinate space that contains this path. We developed a two-variable analytic representation that comes close to describing such a surface. Recall that we earlier introduced the matrices $\mathrm{R}_{A}$ and $\mathrm{R}_{D}$ which generate the orientations of the acceptor $\mathrm{A}$ and donor $\mathrm{D}$, starting from a reference geometry $\mathrm{X}_{0}$. Rotation of the acceptor by $\mathrm{R}_{y}(\pi) \mathrm{R}_{D} \mathrm{R}_{A}^{-1}$ and of the donor by $R_{y}(\pi) R_{A} R_{D}^{-1}$ about their respective centers of mass leads to an equivalent equilibrium structure with the roles of acceptor and donor interchanged. The rotation around the $y$-axis over $\pi$ is necessary because the rotation of the acceptor and the donor does not interchange their positions. The matrix $\mathrm{R}_{y}(\pi) \mathrm{R}_{A} \mathrm{R}_{D}^{-1}$ being orthogonal, it may be expressed as a rotation around a certain axis $\boldsymbol{n}_{D}$ over an angle $\chi_{D} \cdot{ }^{44}$ Similarly the acceptor rotation $\mathrm{R}_{y}(\pi) \mathrm{R}_{D} \mathrm{R}_{A}^{-1}$ is equivalent to a rotation around $\boldsymbol{n}_{A}$ over $\chi_{A}$. The rotation angle $\chi$ is determined by the trace of the orthogonal matrix and thus $\chi_{\mathrm{A}}=\chi_{D}=\chi$. It is not difficult to derive that

$$
\begin{aligned}
\boldsymbol{n}_{A}=\left[\cos \left(\beta_{A}\right)+\frac{\sin \left(\beta_{\mathrm{A}}\right) \sin (\Delta \beta)}{1-\cos (\Delta \beta)},-1, \sin \left(\beta_{A}\right)-\right. \\
\left.\frac{\cos \left(\beta_{\mathrm{A}}\right) \sin (\Delta \beta)}{1-(\Delta \beta)}\right]
\end{aligned}
$$

$$
\begin{aligned}
\boldsymbol{n}_{D}=\left[-\cos \left(\beta_{D}\right)-\frac{\sin \left(\beta_{D}\right) \sin (\Delta \beta)}{1-(\Delta \beta)},\right. & -1, \sin \left(\beta_{D}\right)- \\
& \left.\frac{\cos \left(\beta_{D}\right) \sin (\Delta \beta)}{1-(\Delta \beta)}\right]
\end{aligned}
$$

with $\Delta \beta=180^{\circ}-\beta_{D}-\beta_{A}=-62.18^{\circ}$ and $\chi=105.47^{\circ}$. Numerically, the normalized rotation axes are $\boldsymbol{n}_{A}=(-0.888$, $-0.459,-0.040)$ and $\boldsymbol{n}_{D}=(0.888,-0.459,0.040)$. We now describe the donor-acceptor interchange analytically as a simultaneous (geared) rotation of $\mathrm{A}$ and $\mathrm{D}$ around their respective axes over angles $\chi_{A}$ and $\chi_{D}$, which run from $0^{\circ}$ to $105.47^{\circ}$, see Figure $4 \mathrm{~b}$. The two-dimensional cuts of the tunneling wave functions illustrate their dependence on both $\chi_{A}$ and $\chi_{D}$.

Bifurcation tunneling, see Figure 2, can be represented as a simultaneous increase of $\beta_{A}$ and $\beta_{D}$ (Figure $\left.4 \mathrm{c}\right)^{53}$ and we plot the wave function dependence on both these coordinates.

\subsection{Results}

Tunneling. Figures 5 and 6 show the wave functions in the tunneling regions defined in Figure 4. The largest level splitting occurs as a result of acceptor tunneling, see Figures 2 and 3. This process is clearly visible in Figures $5 \mathrm{a}$ and $5 \mathrm{~b}$ : the $A_{1}^{+}$ and $B_{1}^{+}$wave functions do not possess a node between the two equivalent potential minima shown in Figure $4 \mathrm{a}$, whereas the $A_{2}^{-}$and $B_{2}^{-}$wave functions have a nodal plane between these minima that is nearly orthogonal to the acceptor tunneling path. This confirms that acceptor tunneling indeed occurs by a concerted motion in the acceptor wagging coordinate $\beta_{A}$ and the torsional coordinate $\alpha$.

The donor-acceptor interchange tunneling leading to the smaller splittings between the $A_{1}^{+}$and $B_{1}^{+}$levels and between the $A_{2}^{-}$and $B_{2}^{-}$levels is illustrated in Figure 5 , parts $\mathrm{c}$ and $\mathrm{d}$. The $A_{1}^{+}$and $A_{2}^{-}$wave functions do not show a node, whereas the $B_{1}^{+}$and $B_{2}^{-}$wave functions have a nodal plane in the angles $\chi_{A}$ and $\chi_{B}$ nearly orthogonal to the tunneling path of Figure $4 \mathrm{~b}$. We may conclude that these angles, the construction of which is described in section 3.1, present a clear view of the interchange tunneling process.

As explained in ref 21, bifurcation tunneling does not lead to a further splitting of the energy levels. In accordance with this finding, we observe in Figure 5e that none of the wave functions belonging to the different $G_{16}$ irreps has a node in the angles $\beta_{D}$ and $\beta_{A}$ which vary along the bifurcation tunneling path of Figure $4 \mathrm{c}$.

We noticed in ref 21 that the tunneling level splittings are generally larger in the vibrationally excited states than in the ground state. In some cases, as for example, for the acceptor splitting in the donor torsion (DT) excited state, this is a very pronounced effect. Plots of the wave functions of the vibrationally excited levels are discussed below but because of space limitations the tunneling behavior of these vibrationally excited wave functions is not explicitly displayed. Let us note here that we observed a marked increase of amplitude of the excited wave functions in the tunneling regions, relative to the ground-state wave functions, in good correspondence with the increased tunneling level splittings.

When comparing the wave functions of $\left(\mathrm{D}_{2} \mathrm{O}\right)_{2}$ in Figure 6, parts a-e, with those of $\left(\mathrm{H}_{2} \mathrm{O}\right)_{2}$ in Figure $5, \mathrm{a}-\mathrm{e}$, we note that the former are clearly more localized, because of the larger mass and smaller rotational constants of $\mathrm{D}_{2} \mathrm{O}$. Particularly, the amplitudes of the wave functions in the tunneling regions are 

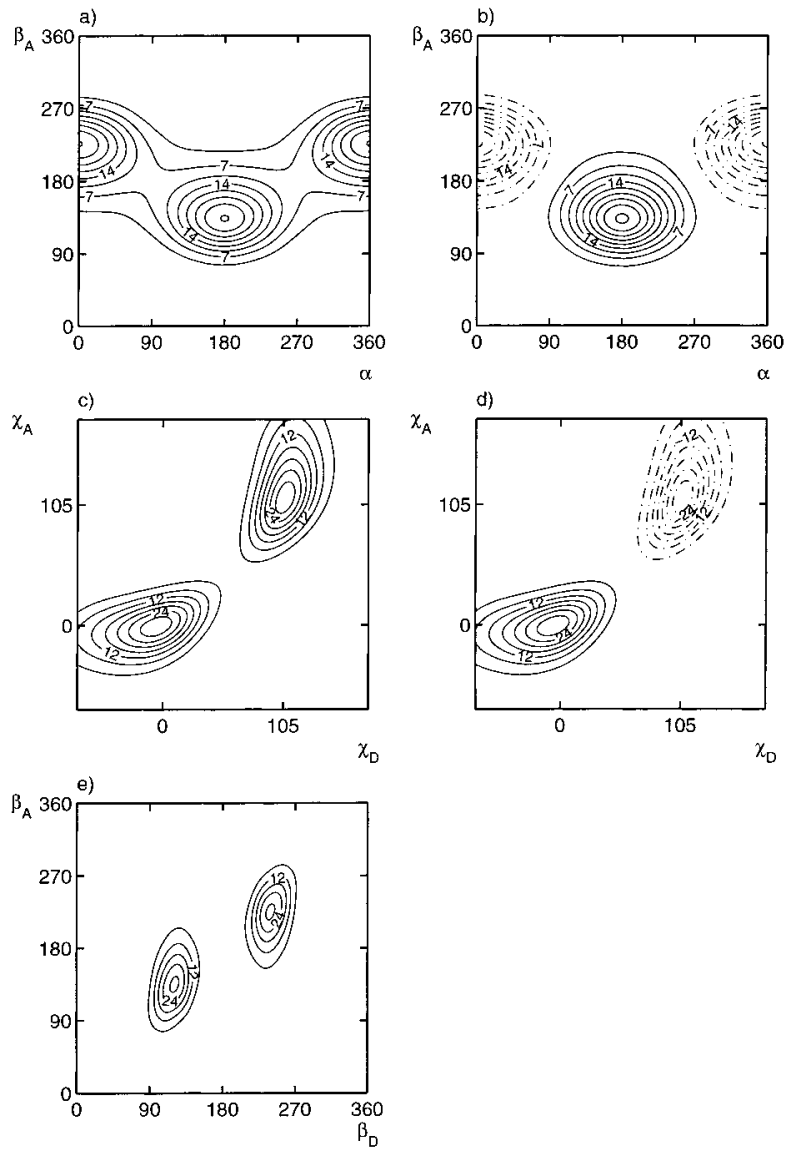

Figure 5. Tunneling wave functions of the $\mathrm{H}_{2} \mathrm{O}$ dimer $(0,0,0)\left(A^{\prime}\right)$ states. (a) Acceptor tunneling of $B_{1}^{+}$(similar to $A_{1}^{+}$); (b) Acceptor tunneling of $B_{2}^{-}$(similar to $A_{2}^{-}$); (c) Interchange tunneling of $A_{2}^{-}$(similar to $A_{1}^{+}$); (d) Interchange tunneling of $B_{2}^{-}$(similar to $B_{1}^{+}$); (e) Bifurcation tunneling of $B_{2}^{-}$(similar to $A_{1}^{+}, B_{1}^{+}$, and $A_{2}^{-}$). The amplitudes (in $a_{0}^{-3 / 2}$ ) are multiplied by a factor of 1000 .

markedly smaller in the $\mathrm{D}_{2} \mathrm{O}$ dimer than in the $\mathrm{H}_{2} \mathrm{O}$ dimer, in agreement with the much smaller energy level splittings in the former system.

Intermolecular Vibrations. Before discussing the exact wave functions of the vibrationally excited states, we present the harmonic frequencies and normal modes computed from the same SAPT-5st potential. They are listed in Tables 7 and 8 for $\left(\mathrm{H}_{2} \mathrm{O}\right)_{2}$ and $\left(\mathrm{D}_{2} \mathrm{O}\right)_{2}$, respectively. We observed already in ref 21 that the harmonic frequencies of the lower intermolecular modes in $\left(\mathrm{H}_{2} \mathrm{O}\right)_{2}$ are typically between $20 \%$ and $40 \%$ larger than those of the corresponding frequencies from nearly exact anharmonic six-dimensional variational calculations. The latter were in very good agreement with the experimental data of Braly et al. ${ }^{32-34}$ Also for the highest observed mode at a frequency of $104 \mathrm{~cm}^{-1}$ in $\left(\mathrm{D}_{2} \mathrm{O}\right)_{2}$, assigned by Braly et al. as in-plane bend (IB), we found very good agreement between the exactly calculated frequency and the experimental value. The harmonic frequency of the IB mode is higher by a factor of 2.6, however, and we provisionally concluded in ref 21 that this mode was incorrectly assigned. After discussing the character of the exact vibrational wave functions, we will come back to this point. Here, we state already that, with the qualifications given below, the assignment of the donor torsion (DT), acceptor wag (AW), and acceptor twist (AT) modes was correct. Comparison of the results in Tables 7 and 8 with the exact frequencies in Tables 3 and 4 shows that the harmonic frequencies of these modes, and also of the stretch (S) mode, are indeed too high by 20 to
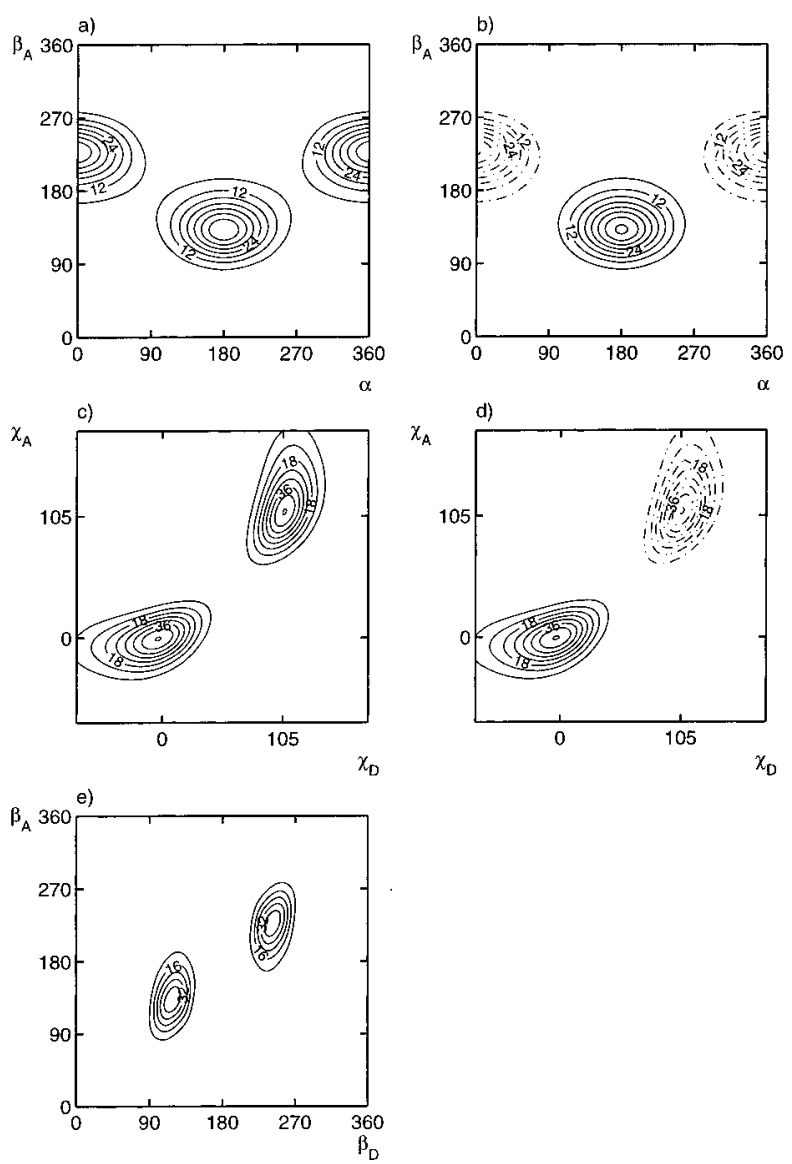

Figure 6. Tunneling wave functions of the $\mathrm{D}_{2} \mathrm{O}$ dimer $(0,0,0)\left(A^{\prime}\right)$ states, see caption of Figure 5.

TABLE 7: Harmonic Frequencies (in $\mathrm{cm}^{-1}$ ) and Displacements of the Internal Coordinates for the Normal Modes of the $\mathrm{H}_{2} \mathrm{O}$ Dimer Calculated from the SAPT-5st Potential

\begin{tabular}{lrcrcrr}
\hline mode & DT $\left(A^{\prime \prime}\right)$ & AT $\left(A^{\prime \prime}\right)$ & AW $\left(A^{\prime}\right)$ & S $\left(A^{\prime}\right)$ & IB $\left(A^{\prime}\right)$ & OB $\left(A^{\prime \prime}\right)$ \\
\hline freq & 121.01 & 143.70 & 157.87 & 186.83 & 369.99 & 564.70 \\
\hline$\alpha(\mathrm{rad})$ & 1.1878 & 0.3466 & 0.0000 & 0.0000 & 0.0000 & 1.1544 \\
$\beta_{A}(\mathrm{rad})$ & 0.0000 & 0.0000 & -0.7966 & 0.5882 & 0.7861 & 0.0000 \\
$\gamma_{A}(\mathrm{rad})$ & -0.3214 & 0.8857 & 0.0000 & 0.0000 & 0.0000 & 0.4617 \\
$\beta_{D}(\mathrm{rad})$ & 0.0000 & 0.0000 & -0.3826 & 0.2521 & -0.5928 & 0.0000 \\
$\gamma_{D}(\mathrm{rad})$ & 0.0073 & 0.5179 & 0.0000 & 0.0000 & 0.0000 & -1.0267 \\
$R\left(a_{0}\right)$ & 0.0000 & 0.0000 & -0.3636 & -0.5139 & 0.0161 & 0.0000
\end{tabular}

TABLE 8: Harmonic Frequencies (in $\mathrm{cm}^{-1}$ ) and Displacements of the Internal Coordinates for the Normal Modes of the $\mathrm{D}_{2} \mathrm{O}$ Dimer Calculated from the SAPT-5st Potential

\begin{tabular}{lrcrrrr}
\hline mode & DT $\left(A^{\prime \prime}\right)$ & AT $\left(A^{\prime \prime}\right)$ & AW $\left(A^{\prime}\right)$ & $\mathrm{S}\left(A^{\prime}\right)$ & $\mathrm{IB}\left(A^{\prime}\right)$ & $\mathrm{OB}\left(A^{\prime \prime}\right)$ \\
\hline freq & \multicolumn{1}{c}{88.07} & 102.87 & \multicolumn{1}{c}{118.52} & 172.84 & 274.76 & \multicolumn{1}{c}{406.11} \\
\hline$\alpha(\mathrm{rad})$ & -0.8743 & 0.2065 & 0.0000 & 0.0000 & 0.0000 & -0.8626 \\
$\beta_{A}(\mathrm{rad})$ & 0.0000 & 0.0000 & -0.6732 & 0.1873 & -0.6367 & 0.0000 \\
$\gamma_{A}(\mathrm{rad})$ & 0.1948 & 0.6485 & 0.0000 & 0.0000 & 0.0000 & -0.3508 \\
$\beta_{D}(\mathrm{rad})$ & 0.0000 & 0.0000 & -0.3345 & 0.1278 & 0.4095 & 0.0000 \\
$\gamma_{D}(\mathrm{rad})$ & -0.0342 & 0.3743 & 0.0000 & 0.0000 & 0.0000 & 0.7184 \\
$R\left(a_{0}\right)$ & 0.0000 & 0.0000 & -0.1836 & -0.5677 & 0.0271 & 0.0000
\end{tabular}

$40 \%$, both for $\left(\mathrm{H}_{2} \mathrm{O}\right)_{2}$ and $\left(\mathrm{D}_{2} \mathrm{O}\right)_{2}$. Furthermore, the ordering of the levels is not the same.

The eigenvectors given in Tables 7 and 8 indicate that the character of the normal modes is not as simple as suggested by Reimers and Watts, ${ }^{37}$ and also by Xantheas and Dunning ${ }^{54}$ who presented harmonic frequencies computed by the $a b$ initio 
Møller-Plesset second-order (MP2) method. The character of each normal mode, i.e., the amplitudes of displacement in the six intermolecular coordinates, can be read from Tables 7 and 8. It is not straightforward to compare the different constituents because the displacement coordinates are nonorthogonal, and the amplitudes in $R\left(a_{0}\right)$ and in the angles (radians) are not even in the same units. It is obvious that no mixing is allowed between $A^{\prime}$ and $A^{\prime \prime}$ modes, which are even and odd, respectively, with respect to the plane of reflection of the equilibrium structure. The first $\left(A^{\prime \prime}\right)$ fundamental is indeed predominantly DT $(\alpha)$, but with a nonnegligible admixture of AT $\left(\gamma_{A}\right)$. The second (again $A^{\prime \prime}$ ) fundamental is AT, with a substantial contribution of OB $\left(\gamma_{D}\right)$ and some DT character. The third $\left(A^{\prime}\right)$ fundamental is $\mathrm{AW}\left(\beta_{A}\right)$, with substantial IB $\left(\beta_{D}\right)$ and some $\mathrm{S}$ $(R)$ mixed in. The fourth $\left(A^{\prime}\right)$ fundamental is $\mathrm{S}$ with both some $\mathrm{AW}$ and some IB character. The highest two fundamentals are even more strongly mixed. The fifth $\left(A^{\prime}\right)$ mode should be IB, but actually, the contribution of AW is larger. And the sixth $\left(A^{\prime \prime}\right)$ mode should be OB but it contains a larger contribution of DT and a substantial amount of AT. Yet, if one compares the relative contributions of each displacement coordinate in all of the normal modes, the nomenclature of refs 37 and 54 is not unreasonable. One must realize, however, that each of the normal modes contains a strong mix of all displacements of the appropriate symmetry.

Similar considerations can be given for the exact wave functions. Vibrational $(J=K=0)$ wave functions that are of $A^{\prime}$ symmetry under the point group $C_{s}$ are of $A_{1}^{+}, E^{+}, B_{1}^{+}, A_{2}^{-}$, $E^{-}, B_{2}^{-}$symmetry under the full PI group $G_{16}$, and those that are of $A^{\prime \prime}$ symmetry under $C_{s}$ are of $B_{1}^{-}, E^{-}, A_{1}^{-}, B_{2}^{+}, E^{+}, A_{2}^{+}$ symmetry under $G_{16}$, see Table 1 of ref 21 . Therefore, the vibrational wave functions of $A^{\prime}$ and $A^{\prime \prime}$ symmetry that do not mix in the harmonic approximation, do not mix in the exact calculations either, at least for the one-dimensional $A$ and $B$ irreps for $K=0$. So, we may expect that mixing primarily occurs in the DT $(\alpha)$, AT $\left(\gamma_{A}\right)$, and OB $\left(\gamma_{D}\right)$ coordinates, and in the AW $\left(\beta_{A}\right), \mathrm{S}(R)$, and IB $\left(\beta_{D}\right)$ coordinates.

Plots of the $(J=K=0)$ vibrational wave functions are shown in Figures 7 and 8 . The states are denoted as $(J, K, v)$. For each vibrationally excited state $(v>0)$ we could choose between the different tunneling components. It turned out that, in contrast with the ground state, the tunneling components belonging to different $G_{16}$ irreps have a slightly different appearance in the vibrational coordinates. We mostly selected the $A_{1}^{+}$component for the $A^{\prime}$ vibrations and the $A_{2}^{+}$component for the $A^{\prime \prime}$ vibrations. In two cases, $(0,0,2)\left(A^{\prime}\right)$ and $(0,0,6)\left(A^{\prime \prime}\right)$, we preferred to plot the $B_{2}^{-}$and $B_{1}^{-}$components because they display more clearly the nodal character of the wave functions. For the $K=$ 1 states we always investigated the $B_{1}^{-}$components.

If the lowest excited $(0,0,1)\left(A^{\prime \prime}\right)$ vibrational state in Figures $7 \mathrm{a}$ and $8 \mathrm{a}$ were of pure DT character, the nodal plane would be perpendicular to the $\alpha$ axis at $\alpha=180^{\circ}$. Instead, one observes a substantial amount of AT $\left(\gamma_{A}\right)$ admixture. Similarly, if the third excited vibrational state $(0,0,3)\left(A^{\prime \prime}\right)$ were pure AT, the nodal plane in Figures $7 \mathrm{~b}$ and $8 \mathrm{~b}$ would be perpendicular to the $\gamma_{A}$ axis, at $\gamma_{A}=90^{\circ}$. Here, one observes considerable DT admixture. In addition, the $(0,0,3)$ state has some $\mathrm{OB}$ character, which may be seen in Tables 5 and 6 by the fact that the maximum and minimum in the wave function are displaced in $\gamma_{D}$ with respect to the equilibrium value of this angle (by about $15^{\circ}$ ). It is noteworthy that the nodal planes in Figure 7 , parts a and $\mathrm{b}$, and in Figure 8, parts a and b, although not horizontal and vertical, are nearly orthogonal, in accordance with the orthogonality of the two vibrational wave functions.
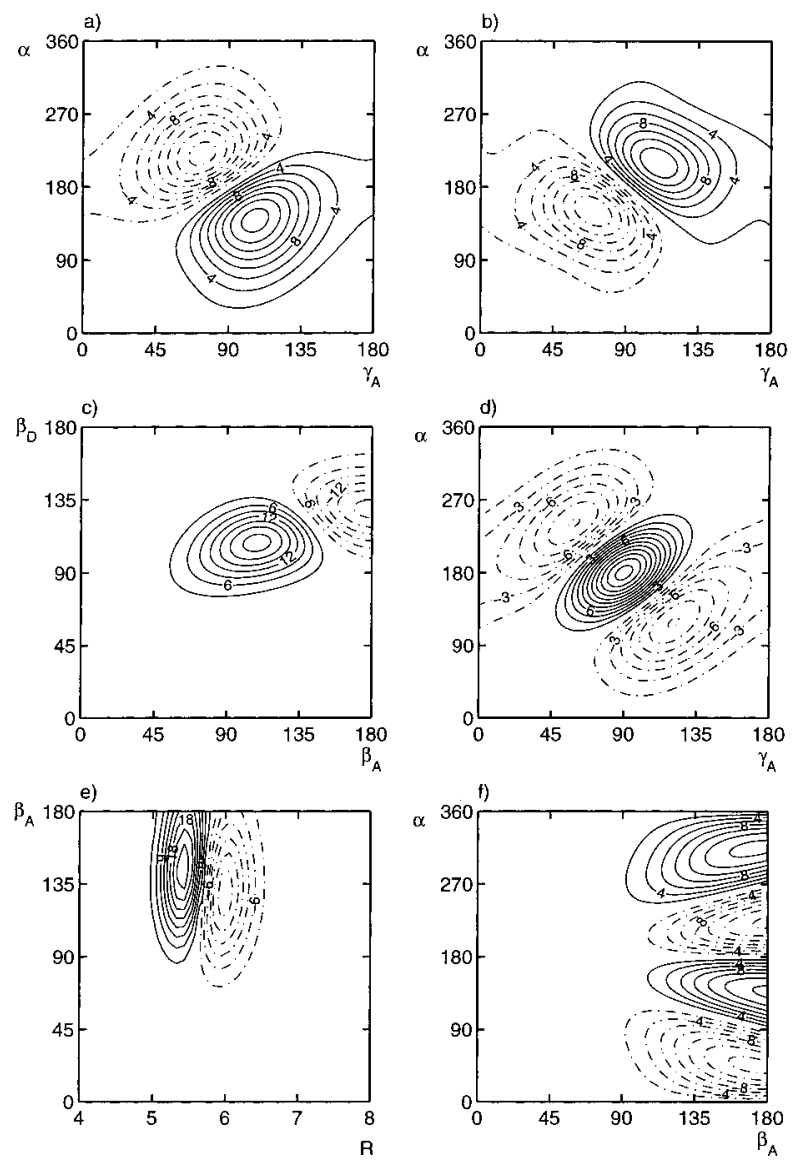

Figure 7. Vibrational wave functions of the $\mathrm{H}_{2} \mathrm{O}$ dimer. (a) $(0,0,1)$ (A") $A_{2}^{+}$; (b) $(0,0,3)\left(A^{\prime \prime}\right) A_{2}^{+}$; (c) $(0,0,2)\left(A^{\prime}\right) B_{2}^{-}$; (d) $(0,0,4)\left(A^{\prime}\right) A_{1}^{+}$; (e) $(0,0,5)\left(A^{\prime}\right) A_{1}^{+}$. (f) $(0,0,6)\left(A^{\prime \prime}\right) B_{1}^{-}$; The amplitudes (in $a_{0}^{-3 / 2}$ ) are multiplied by a factor of 1000 .

The lowest state of $A^{\prime}$ symmetry, $(0,0,2)\left(A^{\prime}\right)$, is not pure AW but has an IB contribution (Figures $7 \mathrm{c}$ and $8 \mathrm{c}$ ). Figures $7 \mathrm{e}$ and 8e illustrate that the $(0,0,5)\left(A^{\prime}\right)$ state corresponds to a stretch vibration. This is confirmed by the comparatively large $\Delta R$ values (0.3403 and $0.2493 a_{0}$, respectively) and shifts in $\langle R\rangle$ in Tables 3 and 4 . Figure $8 \mathrm{e}$ shows that in the case of $\left(\mathrm{D}_{2} \mathrm{O}\right)_{2}$ some AW contributes to this mode. Hence, the DT, AW, AT, and S modes are in qualitative agreement with the normal-mode analysis, even though they have large amplitudes and a substantial degree of anharmonic character.

More surprising is the analysis of the $(0,0,4)\left(A^{\prime}\right)$ state, which in ref 21 was provisionally assigned to the IB vibration, following the assignment ${ }^{32-34}$ of the experimental data. A nodal plane in the wave function along the IB coordinate $\beta_{D}$ could not be discovered, however. Figure $7 d$ shows beyond any doubt that this state actually corresponds to a DT overtone. Just as the DT fundamental shown in Figure 7a, it contains a substantial contribution of AT $\left(\gamma_{A}\right)$ excitation. The fact that the excitation frequency of the $(0,0,4)\left(A^{\prime}\right)$ state is nearly twice the frequency of the lowest tunneling component of the DT fundamental $(0,0,1)\left(A^{\prime \prime}\right)$ supports this conclusion. It holds for both $\left(\mathrm{H}_{2} \mathrm{O}\right)_{2}$ and $\left(\mathrm{D}_{2} \mathrm{O}\right)_{2}$.

Another interesting result emerges from the analysis of the wave function of the $(0,0,6)\left(A^{\prime \prime}\right)$ state. This excited state may be classified as a combination of the mixed DT/AT $\left(\alpha / \gamma_{A}\right)$ fundamental and the AW $\left(\beta_{A}\right)$ mode, see Figures $7 \mathrm{f}$ and $8 \mathrm{f}$. The data shown in Tables 5 and 6 confirm this assignment. The AW contribution can be recognized from the fact that the $\beta_{A}$ value of the maximum in the wave function (about $160^{\circ}$ ) is consider- 

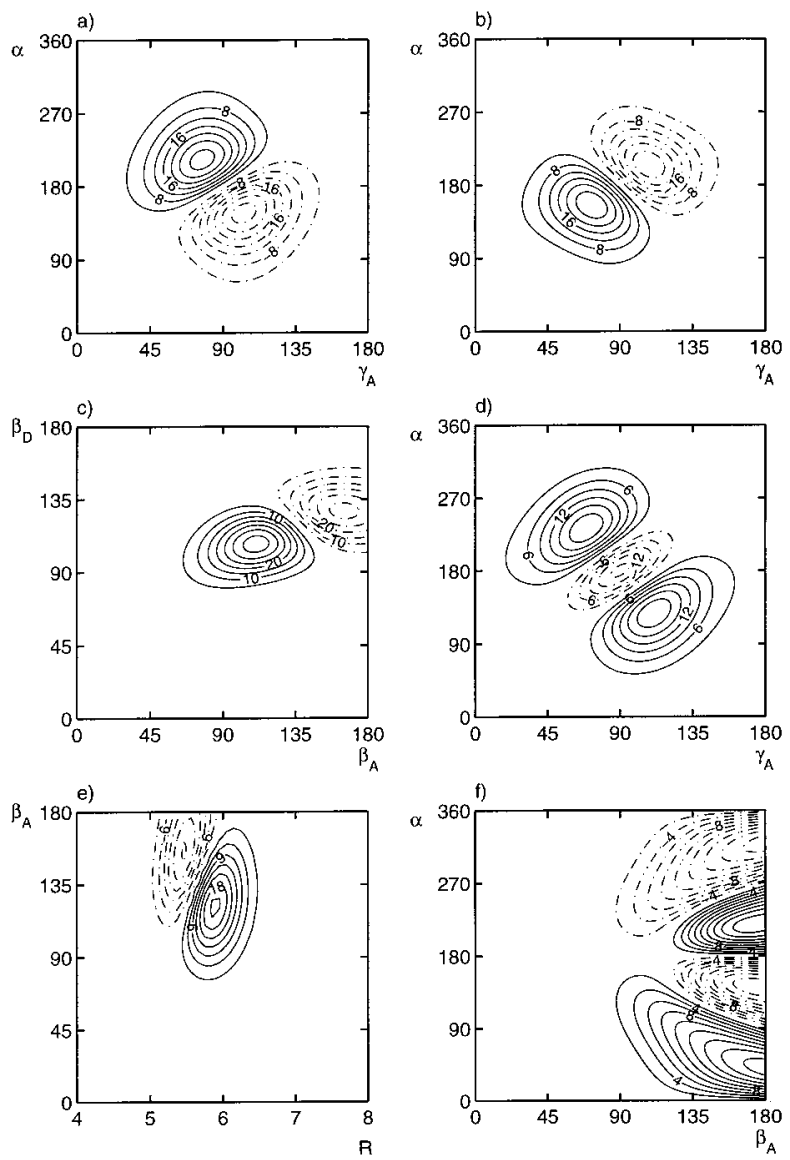

Figure 8. Vibrational wave functions of the $\mathrm{D}_{2} \mathrm{O}$ dimer, see caption of Figure 7.

ably larger than the $\beta_{A}$ value of the maximum in the groundstate wave function (about $130^{\circ}$ ), which is close to the equilibrium value of $\beta_{A} \approx 125^{\circ}$. The assignment of the $(0,0,6)$ $\left(A^{\prime \prime}\right)$ state as a combination of DT/AT and AW holds both for $\left(\mathrm{H}_{2} \mathrm{O}\right)_{2}$ and $\left(\mathrm{D}_{2} \mathrm{O}\right)_{2}$ but with somewhat differently weighted contributions. Moreover, this state has some stretch character in $\left(\mathrm{H}_{2} \mathrm{O}\right)_{2}$, as is evident from the relatively large value of $\Delta R$ $=0.3190 a_{0}$, see Table 3. Figures $7 \mathrm{f}$ and $8 \mathrm{f}$ were plotted with $\beta_{D}$ values taken from Tables 5 and 6 in order to obtain a clearer view of the wave function. We note, however, that for this state, in which the excitation depends to a similar extent on three different angles, a two-dimensional plot is only of limited value.

With the assumed IB band being assigned as the DT overtone band in our exact six-dimensional calculations, one may wonder whether we could identify the actual IB (and OB) vibrations. Our calculations are presently limited to vibrational levels up to about $200 \mathrm{~cm}^{-1}$ above the ground state. Taking into account that the harmonic model overestimates the frequencies by 20 to $40 \%$, we may expect the IB mode to have a frequency of about $250 \mathrm{~cm}^{-1}$ and the $\mathrm{OB}$ mode of about $400 \mathrm{~cm}^{-1}$ (for $\left.\left(\mathrm{H}_{2} \mathrm{O}\right)_{2}\right)$. Even if we would know all the exact wave functions in the proper energy range, it is questionable whether we could indentify these modes. We saw already from the harmonic calculations that there are no pure IB and OB modes. In the exact anharmonic calculations we expect, moreover, a strong coupling with the various overtone and combination states that occur at such high energies.

Upon investigation of the states with $J>0$ and $|K| \geq 0$ we always found-not unexpectedly-that the internal part of the wave functions is very nearly $J$-independent. A surprise arose, however, when we analyzed the vibrational wave functions of different $K$, which appeared to be strongly $K$ dependent. In the presentation of the calculated intermolecular vibrations in Tables 5 and 6 and Figures 5 and 6 of ref 21, and also in the assignment ${ }^{32-34}$ of the experimental data, it was assumed that the vibrationally excited states with $|K|=1$ have nearly the same mode character as the levels with $K=0$ which are close to them in energy. The present analysis shows that this is not always true, however. The $(1,1,1)\left(A^{\prime \prime}\right)$ state, just as the corresponding state with $J=K=0$, is a mixture of DT with AT. The angles corresponding to the $(1,1,1)\left(A^{\prime \prime}\right)$ state in Tables 5 and 6 are similar to those of the $(0,0,1)\left(A^{\prime \prime}\right)$ state. The subsequent $(1,1,2)\left(A^{\prime}\right)$ state is similar to the $(0,0,2)\left(A^{\prime}\right)$ state and corresponds to the AW mode. However, from there on, the character of the $|K|=1$ states deviates considerably from the $K=0$ states. The $(1,1,3)$ state corresponds to a vibration of $C_{s}$ symmetry $A^{\prime}$, for example, whereas the $(0,0,3)$ state has symmetry $A^{\prime \prime}$. Hence, the $(1,1,3)$ state cannot be the AT excited state, as the $(0,0,3)$ state is. Furthermore, the $(1,1,4)\left(A^{\prime}\right)$ state of $\left(\mathrm{H}_{2} \mathrm{O}\right)_{2}$ corresponds to a stretch mode, in contrast with the $(0,0,4)\left(A^{\prime}\right)$ state which is a DT overtone. This is obvious from the large $\Delta R$ value $(0.3345$ $\left.a_{0}\right)$ of the $(1,1,4)\left(A^{\prime}\right)$ state in Table 3 . For $\left(\mathrm{D}_{2} \mathrm{O}\right)_{2}$, on the other hand, the $(1,1,4)$ state is a combination of DT/AT and AW and, therefore, resembles the $(0,0,6)\left(A^{\prime \prime}\right)$ state (Table 6$)$. The $(1,1,5)$ $\left(A^{\prime \prime}\right)$ state of $\left(\mathrm{H}_{2} \mathrm{O}\right)_{2}$ has mainly AT character, see Table 5, whereas the $(0,0,5)\left(A^{\prime}\right)$ state is stretch excited and the $(0,0,6)$ $\left(A^{\prime \prime}\right)$ state is a combination of DT/AT and AW. The $(1,1,5)\left(A^{\prime}\right)$ state of $\left(\mathrm{D}_{2} \mathrm{O}\right)_{2}$ is predominantly a stretch vibration with a large $\Delta R=0.2705 a_{0}$ (Table 4$)$, just as the $(0,0,5)$ state.

This observation, that several of the vibrational levels with $|K|=1$ have a mode character that differs substantially from the nearby levels with $K=0$, explains why the calculated $|K|$ $=1$ minus $K=0$ energy differences are rather irregular. Normally, one can extract the ground and excited-state rotational constants $A$ from these differences. We noted already in ref 21 that the excited-state constants $A$ which were thus obtained looked anomalous and, in fact, reflect the internal motions of the dimer.

Finally, we observe that, as expected, the vibrational states are more localized in $\left(\mathrm{D}_{2} \mathrm{O}\right)_{2}$ than in $\left(\mathrm{H}_{2} \mathrm{O}\right)_{2}$. This is obvious from the wave function plots in Figures 7 and 8, as well as from the $\Delta R$ values in Tables 3 and 4 and from the displacements of the angles in Tables 5 and 6 . The harmonic eigenvectors in Tables 7 and 8 show the same effect.

\section{Transition Moments}

In this section, we study the dipole transitions between different VRT states. To discuss their selection rules, we first recall that the group $G_{16}$, which is isomorphic to the point group $D_{4 h}$, is a direct product group: $G_{16}=\operatorname{PI}\left(D_{4}\right) \otimes P I\left(C_{i}\right)$, with $P I\left(C_{i}\right) \equiv\left\{E, E^{*}\right\}$. We distinguish here the permutation-inversion (PI) group from the corresponding point group by the prefix $P I$. Sometimes ${ }^{49}$ PI groups are designated by the suffix $M$, thus e.g., $P I\left(D_{4}\right) \equiv D_{4}(M)$. The permutation-inversion group $P I\left(D_{4}\right)$ contains pure (unstarred) permutations only. The irreducible $P I\left(D_{4}\right)$ projectors are given in Table 9 . For future reference we note that $P_{A B}$ is the only generator of $G_{16}$ that interchanges the hydrogen and oxygen labels of the monomers. See Table 10 for the action of the generators on the coordinates.

Because the dipole operator $\mu_{m}^{\mathrm{SF}}$ defined in eq 7 is totally symmetric under any permutation, it transforms as $A_{1}$ with respect to $P I\left(D_{4}\right)$ and the dipole selection rule is accordingly $\Gamma$ $\rightarrow \Gamma$, with $\Gamma$ indicating an irrep of $P I\left(D_{4}\right)$. Further, $\mu_{m}^{\mathrm{SF}}$ is antisymmetric under $E^{*}$, so that it belongs to $A_{1}^{-}$of $G_{16}$ and hence only the transitions $\Gamma^{ \pm} \rightarrow \Gamma^{\mp}$ are allowed. The selection 
TABLE 9: Symmetry Projectors onto Irreps $\Gamma$ of $P I\left(D_{4}\right)^{a}$

\begin{tabular}{ccc}
\hline$\Gamma$ & projector & spin \\
\hline$A_{1}$ & $1 / 8\left[E+P_{\mathrm{AB}}\right][E+(34)][E+(12)]$ & $p-p$ \\
$A_{2}$ & $1 / 8\left[E-P_{\mathrm{AB}}\right][E-(34)][E-(12)]$ & $o-o$ \\
$B_{1}$ & $1 / 8\left[E-P_{\mathrm{AB}}\right][E+(34)][E+(12)]$ & $p-p$ \\
$B_{2}$ & $1 / 8\left[E+P_{\mathrm{AB}}\right][E-(34)][E-(12)]$ & $o-o$ \\
$\mathrm{E}_{\mathrm{x}}$ & $1 / 4[E-(34)][E+(12)]$ & $o-p$ \\
$\mathrm{E}_{\mathrm{y}}$ & $1 / 4 P_{\mathrm{AB}}[E-(34)][E+(12)]$ & $p-o$
\end{tabular}

${ }^{a}$ Here $P_{A B} \equiv\left(O_{1} O_{2}\right)(13)(24)$. Projectors onto irreps $\Gamma^{ \pm}$of $G_{16}$ are obtained by multiplication with $\left[E \pm E^{*}\right]$. In the column marked "spin" $o$ and $p$ refer to the ortho and para coupling of the spins of the hydrogen nuclei in each monomer. This assignment is for $\left(\mathrm{H}_{2} \mathrm{O}\right)_{2}$; for $\left(\mathrm{D}_{2} \mathrm{O}\right)_{2} O$ and $p$ must be interchanged. $E_{x}$ and $E_{y}$ are partners in the twodimensional irrep $E$.

TABLE 10: Action of the $G_{16}$ Generators on the Coordinates $^{a}$

\begin{tabular}{lllll}
\hline$E$ & $(12)$ & $(34)$ & $\mathrm{P}_{\mathrm{AB}}$ & \multicolumn{1}{c}{$E^{*}$} \\
\hline$\Phi$ & $\Phi$ & $\Phi$ & $\Phi+\pi$ & $\Phi+\pi$ \\
$\Theta$ & $\Theta$ & $\Theta$ & $\pi-\Theta$ & $\pi-\Theta$ \\
$\alpha^{D}$ & $\alpha^{D}$ & $\alpha^{D}$ & $-\alpha^{A}$ & $\pi-\alpha^{D}$ \\
$\beta^{D}$ & $\beta^{D}$ & $\beta^{D}$ & $\pi-\beta^{A}$ & $\beta^{D}$ \\
$\gamma^{D}$ & $\gamma^{D}$ & $\pi+\gamma^{D}$ & $\pi+\gamma^{A}$ & $-\gamma^{D}$ \\
$\alpha^{A}$ & $\alpha^{A}$ & $\alpha^{A}$ & $-\alpha^{D}$ & $\pi-\alpha^{A}$ \\
$\beta^{A}$ & $\beta^{A}$ & $\beta^{A}$ & $\pi-\beta^{D}$ & $\beta^{A}$ \\
$\gamma^{A}$ & $\pi+\gamma^{A}$ & $\gamma^{A}$ & $\pi+\gamma^{D}$ & $-\gamma^{A}$
\end{tabular}

${ }^{a}$ See Figure 1 for the definition of the Euler angles. The angles $\Theta$ and $\Phi$ are the spherical polar angles of $\boldsymbol{R}_{A B}$ with respect to an arbitrary space-fixed frame

rules on the rotational transitions are simply given by the $3 j$ symbol in the square root of the Hönl-London factor ${ }^{55}$

$$
d_{J^{\prime} J K^{\prime} K}=\left[\left(2 J^{\prime}+1\right)(2 J+1)\right]^{1 / 2}\left(\begin{array}{lll}
J^{\prime} & 1 & J \\
-K^{\prime} & k & K
\end{array}\right)
$$

That is, parallel transitions $(k=0)$ satisfy $|\Delta K|=0$, whereas perpendicular transitions $(|k|=1)$ satisfy $|\Delta K|=1$. Further, $|\Delta J|=0,1$. Recall that the $3 j$ symbol vanishes unless $k=K^{\prime}-$ $K$

The symmetry of the wave functions under the permutations (12) and (34) follows directly from the basis in eq 4. Even/odd quantum numbers $k_{\mathrm{A}}$ and $k_{\mathrm{B}}$ determine whether the functions are symmetric/antisymmetric under (12) and (34). This symmetry is associated with the hydrogen nuclear spins $I_{\mathrm{A}}$ and $I_{\mathrm{B}}$ of monomers A and B. Writing $o$ for ortho $\left(I_{X}=1, k_{X}\right.$ is odd $)$, and $p$ for para $\left(I_{X}=0, k_{X}\right.$ is even $)$, where $\mathrm{X}=\mathrm{A}$ or $\mathrm{B}$, we find for $\left(\mathrm{H}_{2} \mathrm{O}\right)_{2}$ the assignment in Table 9. (Table 2 of ref 21 is incorrect: the spin statistical weights $w_{\left(\mathrm{H}_{2} \mathrm{O}\right)_{2}}$ must be $1,3,0,6$, for $A_{1}^{ \pm}, A_{2}^{ \pm}, B_{1}^{ \pm}$, and $B_{2}^{ \pm}$, respectively. This error has its origin in a similar error for $w_{\left(\mathrm{D}_{2} \mathrm{O}\right)_{2}}$ in Table 5 of ref 56.) For $\mathrm{D}_{2} \mathrm{O}$, ortho $\left(I_{X}=0,2\right)$ is associated with even $k_{X}$, para $\left(I_{X}=1\right)$ with odd $k_{\mathrm{X}}$. Note that the nuclear spin is conserved under dipole transitions, as one would expect.

In Tables 11 and 12, we present a list of moments belonging to transitions between ground vibrational tunneling states $(v=$ 0 ). In Table 13 we find some of the transitions from the $v=0$ states to the excited vibrational states. A complete table of vibrational transitions is provided as Supporting Information (Table 13S). Different values of $J=0,1$ and $K=0,1$ are considered. These tables contain results for $\left(\mathrm{H}_{2} \mathrm{O}\right)_{2}$ as well as for $\left(\mathrm{D}_{2} \mathrm{O}\right)_{2}$. Not all allowed transitions are given because many have equal transition moments. For instance, we do not list parallel $\Delta J=-1$ transitions because $(1,0,0) \rightarrow(0,0, v)$ transition moments are nearly identical to those associated with $(0,0,0)$ $\rightarrow(1,0, v)$ transitions. This follows from eq 8 and the fact that the internal part of the wave function hardly depends on $J$. The internal part of the wave function is the part depending on $R$, $\beta_{D}, \gamma_{D}, \beta_{A}, \gamma_{\mathrm{A}}$, and $\alpha$, cf. section 2 . Note, parenthetically, that no transitions $(0,0,0)\left(A^{\prime}\right) \rightarrow(1,0, v)\left(A^{\prime \prime}\right)$ are listed in Table 13 because these transitions are forbidden. The corresponding selection rule follows from the correlation of the $C_{S}$ irreps $A^{\prime}$ and $A^{\prime \prime}$ with the $G_{16}$ irreps (see Table 1 of ref 21 ) and the fact that the total dipole is of $A_{1}^{-}$symmetry. Out of the perpendicular transitions only those with $|\Delta J|=1$ are selected. The $Q$-type $(\Delta J=0)$ transition moments are to a very good approximation equal-with the exception of the square root of a Hönl-London factor $(3 / 2)^{1 / 2}$ - to the corresponding $P$ - and $R$-type transitions, again because the internal parts of the states $(0,0, v)$ and $(1,0, v)$ are nearly identical.

Analysis of Permanent and Tunneling Transition Dipole Moments. Obviously the water dimer has a large permanent dipole moment. However, all VRT states are of well-defined parity and $\mu_{m}^{\mathrm{SF}}$ is of $A_{1}^{-}$symmetry; hence, the expectation value of the SF dipole moment vanishes with respect to any state. To explain the very existence of the permanent dipole of the water dimer, we must consider the symmetry of the BF dipole, cf. eq 6 . Let us first focus on the parallel $|\Delta K|=k=0$ moments. Their values are given-after contraction over the eigenvector coefficients and integration over the radial functions-by eq 8 . Because $J^{\prime}, J M^{\prime}, M K^{\prime}, K$ and $k=0$ are fixed, we can divide out the rotational or "external" factor that depends on these quantum numbers. Note that this external factor is in essence the square root of the Hönl-London factor given in eq 13. The $3 j$-symbol containing $M^{\prime}$ and $M$, which drops out of the formalism after averaging over all spatial directions, is not included in this Hönl-London factor. The part of the transition dipole moment remaining after this division is referred to as the "internal" (transition or permanent) moment.

The parallel dipole operator, the $k=0$ term in eq 7, has as the "external" part the function $D_{m 0}^{(1)}(\Phi, \Theta, 0) *$. Table 10 shows that the functions $D_{m k}^{(J)}(\Phi, \Theta, 0)^{*}$ are invariant under (12) and (34). The following relations are easily derived

$$
\begin{aligned}
& P_{A B} D_{m, k}^{(J)}(\Phi, \Theta, 0)^{*}=E^{*} D_{m, k}^{(J)}(\Phi, \Theta, 0)^{*}= \\
&(-1)^{J} D_{m,-k}^{(J)}(\Phi, \Theta, 0)^{*}
\end{aligned}
$$

Clearly, the function $D_{m 0}^{(1)}(\Phi, \Theta, 0)^{*}$ is antisymmetric under $E^{*}$ and $P_{A B}$ and is symmetric under the other generators and thus is of $B_{1}^{-}$symmetry for all $m$. This follows by inspection of the projectors in Table 9 . The total dipole being of $A_{1}^{-}$symmetry, the "internal" part $\mu_{0}^{\mathrm{BF}}$ has symmetry $B_{1}^{+}$. In the case of onedimensional irreps, the product of bra and ket in an expectation value has symmetry $A_{1}^{+}$, so that the expectation value of the internal dipole moment vanishes for all states of one-dimensional symmetry species. Because the product $E^{\sigma} \otimes E^{\sigma}(\sigma= \pm)$ contains the $B_{1}^{+}$irrep, ${ }^{57}$ states of $E^{ \pm}$symmetry may have a nonvanishing internal dipole expectation value. As we discussed in the preceding paragraph, this internal dipole may be extracted from the total transition dipole matrix elements $\left\langle(0,0,0)\left(E^{ \pm}\right)\right|$ $\mu_{0}^{\mathrm{SF}}\left|(1,0,0)\left(E^{\mp}\right)\right\rangle$ in the first block of Table 12 by division with the square root $d_{J^{\prime} J K^{\prime} K}$ of a Hönl-London factor; here $d_{1000}=$ -1 . Note that the internal part of the wave function is practically $J$-independent, so that bra and ket in this transition matrix element differ only in the external parts labeled by $J=0$ and 1 , respectively. The internal part of the matrix element is an expectation value that represents the permanent dipole of the water dimer.

To interpret the calculated values of the internal (permanent or transition) moments, we introduce a simple group-theoretical 
TABLE 11: Ground State Tunneling Transition Moments $\mu$ (absolute values in $\left.e a_{0}\right)^{a}$

\begin{tabular}{|c|c|c|c|c|c|c|}
\hline \multirow[b]{2}{*}{ transition } & \multicolumn{3}{|c|}{$\left(\mathrm{H}_{2} \mathrm{O}\right)_{2}$} & \multicolumn{3}{|c|}{$\left(\mathrm{D}_{2} \mathrm{O}\right)_{2}$} \\
\hline & $E_{\mathrm{i}} \rightarrow E_{\mathrm{f}}$ & $\mu$ & $\left(\mu^{(0)}\right)$ & $E_{\mathrm{i}} \rightarrow E_{\mathrm{f}}$ & $\mu$ & $\left(\mu^{(0)}\right)$ \\
\hline$(0,0,0) \rightarrow(1,0,0)$ & & & & & & \\
\hline$A_{1}^{+} \rightarrow A_{1}^{-}$ & $0.00 \rightarrow 1.14$ & 0.934 & $(0.834)$ & $0.00 \rightarrow 0.42$ & 0.893 & $(0.805)$ \\
\hline$B_{1}^{+} \rightarrow B_{1}^{-}$ & $0.72 \rightarrow 0.42$ & 0.934 & (0.834) & $0.04 \rightarrow 0.38$ & 0.893 & $(0.805)$ \\
\hline$A_{2}^{-} \rightarrow A_{2}^{+}$ & $11.21 \rightarrow 12.28$ & 0.903 & $(0.807)$ & $1.69 \rightarrow 2.11$ & 0.884 & (0.797) \\
\hline 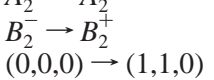 & $11.86 \rightarrow 11.63$ & 0.903 & $(0.807)$ & $1.73 \rightarrow 2.07$ & 0.884 & $(0.797)$ \\
\hline$A_{1}^{+} \rightarrow A_{1}^{-}$ & $0.00 \rightarrow 15.43$ & 0.172 & $(0.185)$ & $0.00 \rightarrow 5.49$ & 0.126 & $(0.134)$ \\
\hline$B_{1}^{+} \rightarrow B_{1}^{-}$ & $0.72 \rightarrow 14.72$ & 0.172 & $(0.184)$ & $0.04 \rightarrow 5.46$ & 0.126 & (0.134) \\
\hline$A_{2}^{-} \rightarrow A_{2}^{+}$ & $11.21 \rightarrow 12.66$ & 0.112 & $(0.120)$ & $1.69 \rightarrow 4.94$ & 0.112 & (0.119) \\
\hline $\begin{array}{l}B_{2}^{-} \rightarrow A_{2}^{+} \\
(1,1,0) \rightarrow(1,1,0)\end{array}$ & $11.86 \rightarrow 12.13$ & 0.112 & $(0.120)$ & $1.73 \rightarrow 4.91$ & 0.112 & (0.119) \\
\hline$A_{1}^{+} \rightarrow A_{1}^{-}$ & $14.72 \rightarrow 15.43$ & 1.042 & $(0.934)$ & $5.46 \rightarrow 5.49$ & 1.086 & (0.979) \\
\hline$B_{1}^{+} \rightarrow B_{1}^{-}$ & $15.43 \rightarrow 14.72$ & 1.042 & $(0.934)$ & $5.49 \rightarrow 5.46$ & 1.086 & (0.979) \\
\hline$A_{2}^{-} \rightarrow A_{2}^{+}$ & $12.13 \rightarrow 12.66$ & 1.051 & $(0.942)$ & $4.91 \rightarrow 4.94$ & 1.089 & (0.982) \\
\hline$B_{2}^{-} \rightarrow B_{2}^{+}$ & $12.66 \rightarrow 12.13$ & 1.051 & $(0.942)$ & $4.94 \rightarrow 4.91$ & 1.089 & $(0.982)$ \\
\hline
\end{tabular}

${ }^{a}$ Energies $E_{i}$ and $E_{f}$ in $\mathrm{cm}^{-1}, \mu$ includes the induced dipole moments, $\mu^{(0)}$ only the permanent dipoles of the monomers. The $3 j$ symbol depending on the "external" quantum numbers $M^{\prime}$ and $M$ in eq 8 is omitted from the calculated values of $\mu$, because it does not explicitly appear in the line strengths.

TABLE 12: Ground State Rotational and Tunneling Transition Moments (in $e a_{0}$ ) of $E^{ \pm}$States. For Explanations, See Table 11

\begin{tabular}{|c|c|c|c|c|c|c|}
\hline \multirow[b]{2}{*}{ transition } & \multicolumn{3}{|c|}{$\left(\mathrm{H}_{2} \mathrm{O}\right)_{2}$} & \multicolumn{3}{|c|}{$\left(\mathrm{D}_{2} \mathrm{O}\right)_{2}$} \\
\hline & $E_{\mathrm{i}} \rightarrow E_{\mathrm{f}}$ & $\mu$ & $\left(\mu^{(0)}\right)$ & $E_{\mathrm{i}} \rightarrow E_{\mathrm{f}}$ & $\mu$ & $\left(\mu^{(0)}\right)$ \\
\hline$(0,0,0) \rightarrow(1,0,0)$ & & & & & & \\
\hline$E^{+} \rightarrow E^{-}$ & $0.41 \rightarrow 0.83^{a}$ & 0.926 & $(0.833)$ & $0.01 \rightarrow 0.38^{a}$ & 0.892 & $(0.804)$ \\
\hline $\begin{array}{l}E^{-} \rightarrow E^{+} \\
(0,0,0) \rightarrow(1,1,0)\end{array}$ & $11.58 \rightarrow 12.00^{a}$ & 0.897 & $(0.807)$ & $1.71 \rightarrow 2.08^{a}$ & 0.884 & $(0.797)$ \\
\hline$E^{+} \rightarrow E^{-}$ & $0.41 \rightarrow 12.36$ & 0.006 & $(0.007)$ & $0.01 \rightarrow 4.94$ & 0.002 & $(0.002)$ \\
\hline$E^{-} \rightarrow E^{+}$ & $11.58 \rightarrow 12.36$ & 0.113 & $(0.120)$ & $1.71 \rightarrow 4.94$ & 0.111 & (0.119) \\
\hline$E^{+} \rightarrow E^{-}$ & $0.41 \rightarrow 15.05$ & 0.174 & $(0.185)$ & $0.01 \rightarrow 5.49$ & 0.127 & $(0.135)$ \\
\hline $\begin{array}{l}E^{-} \rightarrow E^{+} \\
(1,1,0) \rightarrow(1,1,0)\end{array}$ & $11.58 \rightarrow 15.05$ & 0.003 & $(0.004)$ & $1.71 \rightarrow 5.49$ & 0.0002 & $(0.0003)$ \\
\hline$E^{+} \rightarrow E^{-}$ & $12.36 \rightarrow 12.36^{b}$ & 1.123 & $(1.010)$ & $4.94 \rightarrow 4.94^{b}$ & 1.087 & $(0.980)$ \\
\hline$E^{+} \rightarrow E^{-}$ & $15.05 \rightarrow 15.05^{b}$ & 1.113 & (1.001) & $5.49 \rightarrow 5.49^{b}$ & 1.084 & $(0.977)$ \\
\hline$E^{+} \rightarrow E^{-}$ & $12.36 \rightarrow 15.05$ & 0.080 & (0.072) & $4.94 \rightarrow 5.49$ & 0.022 & $(0.020)$ \\
\hline
\end{tabular}

${ }^{a}$ This is a pure rotational $\Delta J=1$ transition, depending on the permanent (parallel) dipole moment. ${ }^{b}$ This is a transition between two components split by asymmetry doubling; the Coriolis coupling giving the very small splitting between these components was not included.

model in the same spirit as in earlier work on the ammonia dimer. ${ }^{58} \mathrm{We}$ assume that we have two primitive functions, one localized in the donor orientation and denoted by $\psi_{D}(3,4)$ (depending on $\mathrm{O}_{2}$ and protons 3 and 4) and one localized in the acceptor orientation denoted by $\psi_{A}(1,2)$ (depending on $\mathrm{O}_{1}$ and protons 1 and 2). The dependence on the oxygen labels is suppressed in the notation. So, we start from a situation where the hydrogen nuclei 1 and 2 are associated with the acceptor and 3 and 4 with the donor.

Internal functions of $E$ symmetry are projected from the primitive functions with the aid of the projectors in Table 9. After projection, we get the model monomer functions

$$
\begin{aligned}
& |D\rangle \equiv N_{D}[E-(34)]\left[E+E^{*}\right]\left|\psi_{D}(3,4)\right\rangle \\
& |A\rangle \equiv N_{A}[E+(12)]\left[E+E^{*}\right]\left|\psi_{A}(1,2)\right\rangle
\end{aligned}
$$

where 1 and 2 remain on the acceptor $A$ and 3 and 4 on the donor D, i.e., the functions are localized. The constants $N_{\mathrm{D}}$ and $N_{\mathrm{A}}$ are normalization constants. The dimer wave functions of $E^{+}$symmetry become

$$
\begin{gathered}
\left|D A, E_{x}^{+}\right\rangle \equiv|D A\rangle \\
\left|D A, E_{y}^{+}\right\rangle \equiv P_{\mathrm{AB}}|D A\rangle
\end{gathered}
$$

with $|D A\rangle \equiv|D\rangle|A\rangle$. These functions are both localized, although in the second one the labels of the nuclei are interchanged by $P_{A B}$. With the BF dipole operator transforming as $B_{1}^{+}$and, therefore, $P_{A B} \mu_{0}^{\mathrm{BF}} P_{A B}=-\mu_{0}^{\mathrm{BF}}$, we readily derive

$$
\left\langle D A, E_{x}^{+}\left|\mu_{0}^{\mathrm{BF}}\right| D A, E_{x}^{+}\right\rangle=-\left\langle D A, E_{y}^{+}\left|\mu_{0}^{\mathrm{BF}}\right| D A, E_{y}^{+}\right\rangle \neq 0
$$

This expression gives the internal dipole for the $E^{+}$states; the argument is completely analogous for the $E^{-}$states. Writing $\mu_{0}^{\mathrm{BF}}=\mu_{0}^{\mathrm{D}}+\mu_{0}^{\mathrm{A}}$ according to eqs 9 and 10 we see that

$$
\left\langle D A, E_{x}^{+}\left|\mu_{0}^{\mathrm{BF}}\right| D A, E_{x}^{+}\right\rangle=\left\langle D\left|\mu_{0}^{\mathrm{D}}\right| D\right\rangle+\left\langle A\left|\mu_{0}^{\mathrm{A}}\right| A\right\rangle
$$

This result confirms that the expectation values of the dipole with respect to internal states of $E$ symmetry are nonvanishing. In addition we find, because $|D\rangle$ and $|A\rangle$ are localized, that the absolute value of the left-hand side (0.926 $\left.e a_{0}\right)$ must agree reasonably well with the clamped nucleus value of the parallel dipole moment at the equilibrium geometry of the dimer. At this geometry, the sum of permanent plus induced dipole moments is $0.816 e a_{0}$. The difference between these values is due to the averaging over the vibrational ground state, which turns out to increase the dipole moment of the water dimer by about $10 \%$.

Nearly the same internal dipole, but with opposite sign, can be obtained from the $(0,0,0)\left(E^{-}\right) \rightarrow(1,0,0)\left(E^{+}\right)$transition 
TABLE 13: Vibrational Transition Dipole Moments (in $e a_{0}$ ). Explanations, See Table 11

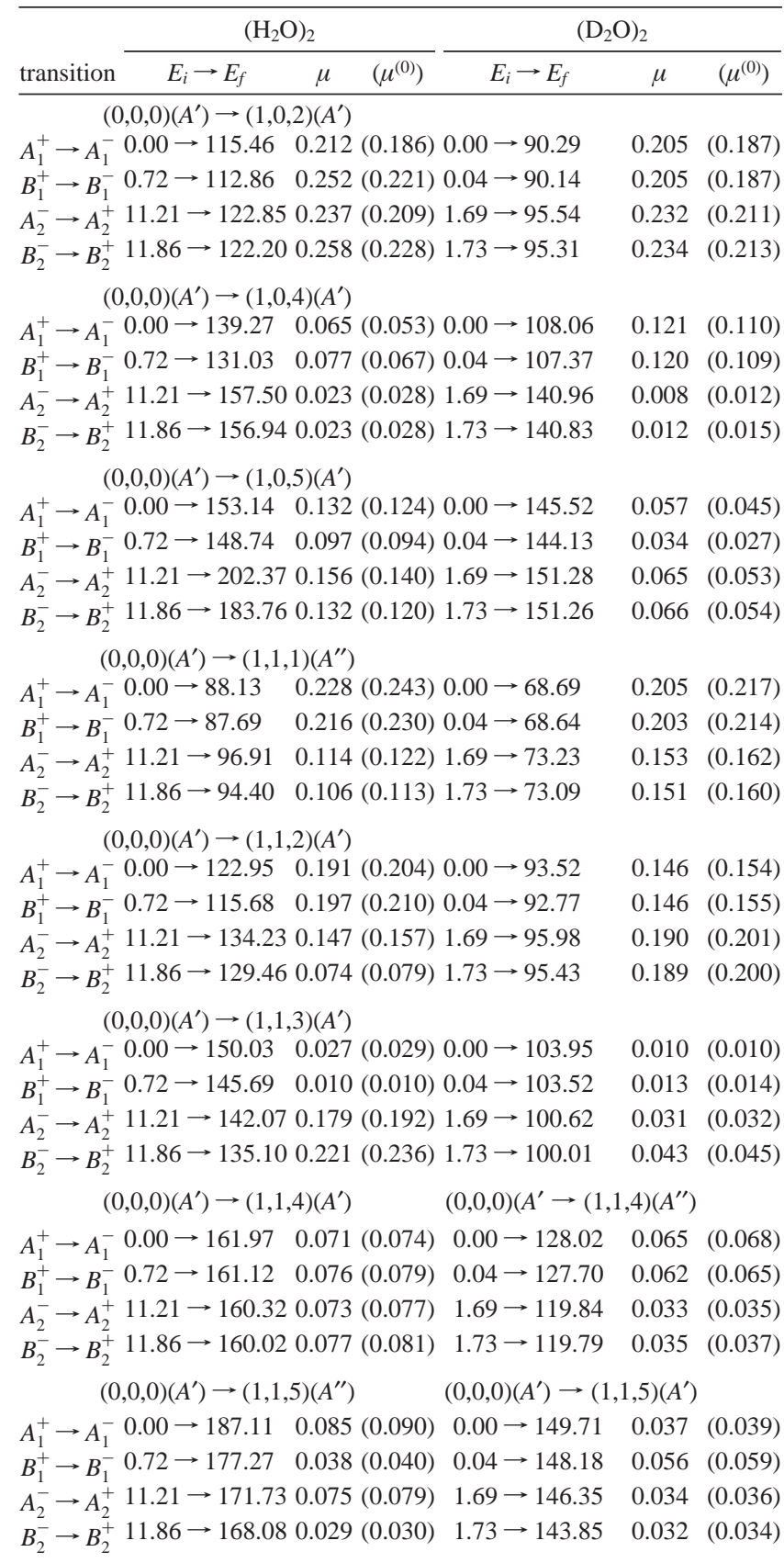

moment in the second line of Table 12. This is again a pure rotational $J=0 \rightarrow 1$ transition, now from the $(0,0,0)\left(E^{-}\right)$state that lies $11.17 \mathrm{~cm}^{-1}$ higher than $(0,0,0)\left(E^{+}\right)$. The $J=0, E_{x}^{ \pm}$ states are plotted in Figure 9. We see that the lower lying $(0,0,0)$ $\left(E^{+}\right)$state is of the kind depicted in Figure 1, that is, monomer $\left\{\mathrm{O}_{2}, \mathrm{H}_{3}, \mathrm{H}_{4}\right\}$ plays the donor and $\left\{\mathrm{O}_{1}, \mathrm{H}_{1}, \mathrm{H}_{2}\right\}$ the acceptor role, whereas for the higher state $(0,0,0)\left(E^{-}\right)$, the roles are reversed. We are considering here the first partner $E_{x}$ in the $E$ irrep, which, as we see in Table 9, is of ortho-para spin. Thus, the energy of the state in which the donor has ortho spin is $11.17 \mathrm{~cm}^{-1}$ more favorable than a state in which the donor has para spin. The acceptor, being the more mobile partner in a hydrogen bonded complex, is less localized in the angles than the donor and hence has a $k_{A}=0$ component, which corresponds to para spin. The donor has odd $k_{\mathrm{D}}$ and is of ortho spin in the energetically most favorable situation.

When comparing the third block of Table 12 with the first block, one observes that the transition dipole moments of parallel
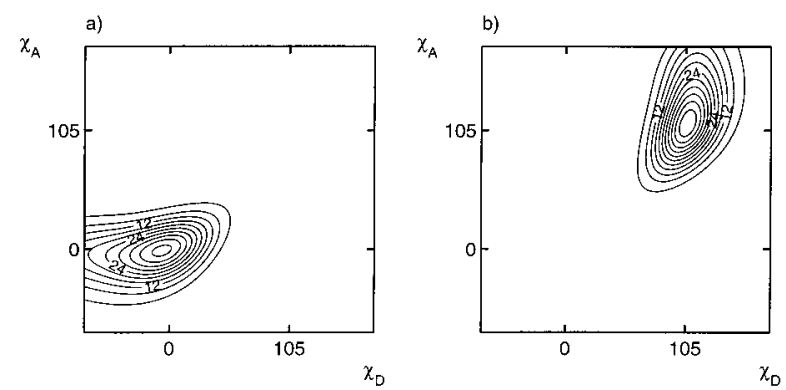

Figure 9. Wave functions of the $\mathrm{H}_{2} \mathrm{O}$ dimer $(0,0,0)\left(A^{\prime}\right)$ states of $E$ symmetry, with $k_{\mathrm{D}}=$ odd and $k_{\mathrm{A}}=$ even (ortho-para). (a) lowest $E^{+}$ state; (b) lowest $E^{-}$state. Notice that these functions are localized at the $\mathrm{DA}$ and $\mathrm{AD}$ geometries for $E^{+}$and $E^{-}$, respectively, in contrast with the corresponding states belonging to the one-dimensional $A$ and $B$ irreps, cf. Figure 5, parts c and d.

transitions between states with $|K|=1$ are about $20 \%$ larger than the parallel transition moments between states with $K=$ 0 . This is due to the larger Hönl-London factor $d_{1111}=3 / \sqrt{6}$ $\approx 1.22$, instead of $d_{1000}=-1$, cf. eq 13 . Hence, the vibrationally averaged internal dipole of the $E^{ \pm}$states with $|K|=1$ is about the same as for $K=0$.

Let us now consider the tunneling transitions in the first block of Table 11. We will show that the transition dipole moments listed in this block are directly related to the size of the permanent dipole of the dimer. The overall rotation function with $J=K=0$ is of $A_{1}^{+}$symmetry and that with $J=1, K=$ 0 of $B_{1}^{-}$symmetry, so that for instance the first transition matrix element

$$
\left\langle(0,0,0)\left(A_{1}^{+}\right)\left|\mu_{m}^{\mathrm{SF}}\right|(1,0,0)\left(A_{1}^{-}\right)\right\rangle=0.934
$$

has an internal matrix element of symmetry $\left\langle A_{1}^{+}\left|\mu_{0}^{\mathrm{BF}}\right| B_{1}^{+}\right\rangle$. We define model monomer functions

$$
\begin{aligned}
& \left.\left|D^{\prime}\right\rangle \equiv N_{D^{\prime}}[E+(34)]\left[E+E^{*}\right] \psi_{D}(3,4)\right\rangle \\
& \left.\left|A^{\prime}\right\rangle \equiv N_{A^{\prime}}[E+(12)]\left[E+E^{*}\right] \psi_{A}(1,2)\right\rangle
\end{aligned}
$$

localized in the donor and acceptor orientation, respectively, with normalization constants $N_{D^{\prime}}$ and $N_{A^{\prime}}$. Because the states belonging to $A_{1}^{+}$and $B_{1}^{+}$are symmetric and antisymmetric under $P_{A B}$, the symmetry adapted internal dimer wave functions are

$$
\begin{aligned}
& \left|D A, A_{1}^{+}\right\rangle \equiv \frac{1}{\sqrt{2}}\left[E+P_{A B}\right]\left|D^{\prime} A^{\prime}\right\rangle \\
& \left|D A, B_{1}^{+}\right\rangle \equiv \frac{1}{\sqrt{2}}\left[E-P_{A B}\right]\left|D^{\prime} A^{\prime}\right\rangle
\end{aligned}
$$

with $\left|D^{\prime} A^{\prime}\right\rangle \equiv\left|D^{\prime}\right\rangle\left|A^{\prime}\right\rangle$. These states are delocalized in the sense that they are linear combinations of DA and AD states (with equal weights $\pm 1 / \sqrt{2}$ ) in which $P_{A B}$ exchanges the labels of the nuclei between $\mathrm{A}$ and $\mathrm{D}$. The internal part of the dipole transition is

$$
\left\langle D A, A_{1}^{+}\left|\mu_{0}^{\mathrm{BF}}\right| D A, B_{1}^{+}\right\rangle=\left\langle D^{\prime} A^{\prime}\left|\mu_{0}^{\mathrm{BF}}\left[E-P_{\mathrm{AB}}\right]\right| D^{\prime} A^{\prime}\right\rangle
$$

Because the differential overlap between $\psi_{D}(3,4) \psi_{A}(1,2)$ and $P_{A B} \psi_{D}(3,4) \psi_{A}(1,2)=\psi_{A}(3,4) \psi_{D}(1,2)$ is very small, cf. Figure 9, we may assume that $\left\langle D^{\prime} A^{\prime}\left|\mu_{0}^{\mathrm{BF}} P_{A B}\right| D^{\prime} A^{\prime}\right\rangle \approx 0$, and we find that the "internal $A_{1}^{+} \rightarrow B_{1}^{+}$transition dipole" actually corresponds to the expectation value $\left\langle D^{\prime} A^{\prime}\left|\mu_{0}^{\mathrm{BF}}\right| D^{\prime} A^{\prime}\right\rangle$. (The dif- 
ferential overlap is not exactly zero, however, otherwise the donor-acceptor interchange tunneling splittings would vanish.) The ket $\left|D^{\prime} A^{\prime}\right\rangle$ is obtained by projection of $\left|\psi_{D}(3,4) \psi_{A}(1,2)\right\rangle$ with operators that do not exchange the labels between the monomers and accordingly is localized. This explains why this transition dipole moment is again close to the clamped nucleus value of the water dimer dipole at the equilibrium geometry DA. Vibrational averaging increases the dipole moment by about $10 \%$, just as we saw for the states of $E^{ \pm}$symmetry. The same argument holds for the other "internal" transition moments in the first block of Table 11.

So far, we have considered parallel transitions only. Turning to the perpendicular transitions, we first consider the $G_{16}$ decomposition of the two-dimensional space carried by the functions $D_{m k}^{(1)}(\Phi, \Theta 0)^{*}, k=-1,1$, appearing in the total dipole moment operator $\mu_{m}^{\mathrm{SF}}$ in eq 7 . In eq 14 , it is stated that effectively $P_{A B}=E^{*}$ in this space. Inspection of the projectors in Table 9 shows then that the external $|k|=1$ functions have a $B_{1}^{-}$and an $A_{1}^{+}$component. From the $A_{1}^{-}$symmetry of $\mu_{m}^{\mathrm{SF}}$ it follows that $\mu_{1}^{\mathrm{BF}}$ and $\mu_{-1}^{\mathrm{BF}}$ have a $B_{1}^{+}$and an $A_{1}^{-}$component. The $A_{1}^{-}$component of $\mu_{ \pm 1}^{\mathrm{BF}}$ gives a nonvanishing transition moment if the symmetry of bra and ket differs only in the \pm parity label of the $G_{16}$ irrep. The $B_{1}^{+}$component connects states of $A$ and $B$ symmetry with the same sub- and superscripts. Neither of these dipole components connects states of $A_{1}^{ \pm}$and $B_{1}^{ \pm}$symmetry with states of $A_{2}^{ \pm}$and $B_{2}^{ \pm}$symmetry. In other words, as one can read off from Table 9, transitions between states belonging to one-dimensional irreps $A$ and $B$ are allowed only if they conserve the symmetry under (12) and (34). It is the symmetry under (12) that is relevant here because (12) corresponds to acceptor tunneling. The conservation of (12) symmetry implies that only transitions occur from the upper prongs of the $K=0$ fork to the lower prongs of the $K=1$ fork, cf. Figure 3, and from the lower prongs of $K=0$ to the upper ones of $K=1$. These transitions are reasonably strong, on the order of 0.15 $e a_{0}$, see Table 11 . The perpendicular $E$ transition moments that satisfy the same "selection rule" (i.e., upper $\leftrightarrow$ lower prongs of different forks) are also of this order of magnitude, but those that break this "selection rule" are much weaker. They are, however, not forbidden. If the frequency of either one of these could be observed, it would give the information needed to fix experimentally the size of the $K=0$ and the $K=1$ acceptor tunneling gaps separately.

Vibrational Transition Moments. All transitions discussed above were tunneling transitions, $\Delta E<20 \mathrm{~cm}^{-1}$. Table 13 gives transitions to vibrationally excited states. The parallel transitions for $K=0$ are between states that are symmetric under $C_{s}\left(A^{\prime}\right.$ $\rightarrow A^{\prime}$ ). The perpendicular and the parallel $K \neq 0$ transitions do not satisfy a selection rule associated with $C_{s}$. The first block of transitions, with $\Delta E \approx 120 \mathrm{~cm}^{-1}$ for $\left(\mathrm{H}_{2} \mathrm{O}\right)_{2}$ and $\Delta E \approx 90$ $\mathrm{cm}^{-1}$ for $\left(\mathrm{D}_{2} \mathrm{O}\right)_{2}$, are acceptor wag $(\mathrm{AW})$ fundamental modes. They are strongly allowed (transition moment $\approx 0.25 e a_{0}$ ). The fourth block $\left(\Delta E \approx 90 \mathrm{~cm}^{-1}\right.$ for $\left(\mathrm{H}_{2} \mathrm{O}\right)_{2}$ and $\Delta E \approx 70 \mathrm{~cm}^{-1}$ for $\left.\left(\mathrm{D}_{2} \mathrm{O}\right)_{2}\right)$ is also strongly allowed. The numbers in this block pertain to the donor torsion (DT) fundamental. The transitions in the second block $\left(\Delta E \approx 145 \mathrm{~cm}^{-1}\right.$ for $\left(\mathrm{H}_{2} \mathrm{O}\right)_{2}$ and $\Delta E \approx$ $120 \mathrm{~cm}^{-1}$ for $\left.\left(\mathrm{D}_{2} \mathrm{O}\right)_{2}\right)$ resemble a DT overtone. These transitions would be strictly forbidden if the intermolecular vibrations were harmonic. This not being the case apparently, they are allowed, although they are about an order of magnitude weaker than the corresponding fundamentals. The third block of transitions gives stretch fundamentals; observe that these are strongly allowed. If the stretch were pure, with no angular modes mixed in, and the dipole moment function would correspond to permanent moments only, the stretch transition would be forbidden. The final vibrational transition that we draw attention to is the transition $(1,1,0)\left(A^{\prime}\right) \rightarrow(0,0,6)\left(A^{\prime \prime}\right)$ given in the supplementary Table 13S. The final states in this transition are (tunneling split) combinations of DT and AW. Harmonically, the transitions are forbidden, but here they are fairly strong, the largest transition moment being $0.114 e a_{0}$. To end the discussion of the vibrational transitions, we like to point out that quite a few of them have been observed ${ }^{33,34}$ for $\left(\mathrm{H}_{2} \mathrm{O}\right)_{2}$, as well as for $\left(\mathrm{D}_{2} \mathrm{O}\right)_{2}$. On the whole, the agreement between experimental and calculated levels is strikingly good. See ref 21 for a detailed comparison of the frequencies.

We end this section by emphasizing that the polarization effects give a nonnegligible contribution to the line strengths. Everywhere in Tables 11-13, we have given the unpolarized transition moments in parentheses. We see that the polarization contributions are on the order of $10 \%$. Because line strengths contain the squared moments, the cross (interference between permanent and induced dipole) terms add about $20 \%$ to the intensity of the lines.

\section{Conclusions}

In this work, we analyzed the nature of the lowest vibrational states of the dimers $\left(\mathrm{H}_{2} \mathrm{O}\right)_{2}$ and $\left(\mathrm{D}_{2} \mathrm{O}\right)_{2}$. The energies of these states are split due to tunneling of the dimer between eight equivalent minima and the corresponding tunneling wave functions are distinguished by their nodal planes. We considered the overall rotational states $J=0$ and $J=1$, with corresponding $|K|=0,1$. Considerable part of the VRT spectrum has been measured and the observed vibrational modes were tentatively assigned to pure modes such as "donor torsion", "acceptor wag", and so forth. Having the VRT wave functions, we are in a position to inspect the nature of these motions. We foundalready at the harmonic level-that none of the modes is pure: all contain a strong mix of all displacements of the appropriate symmetry. In most cases, the earlier assignments can be maintained, however, at least with the qualifications mentioned in section 3.2. That is, in most cases the named mode is present in the state with a nonnegligible weight. There is one exception, though, which is the $(0,0,4)\left(A^{\prime}\right)$ state that earlier ${ }^{32-34}$ was assigned to the in-plane bend (IB) vibration. We found without any doubt that it is a donor torsion (DT) overtone. Also noticeable in this respect is the state $(0,0,6)\left(A^{\prime \prime}\right)$, which is a combination of a donor torsion and an acceptor wag mode.

Another noticeable fact that emerged from our calculations is that the internal states are strongly affected by the value of $|K|$. We already pointed this out in our earlier paper, ${ }^{21}$ where we saw that this strong dependence on $K$ leads to highly irregular values for the rotational constant $A$ of the dimer.

We also presented a set of transition moments, for the main reason that they will help spectroscopists in finding new tunneling and vibrational transitions. One likely candidate of observation is the perpendicular $E^{+} \rightarrow \mathrm{E}^{-}$tunneling transition. For $\left(\mathrm{D}_{2} \mathrm{O}\right)_{2}$, this transition is at $4.93 \mathrm{~cm}^{-1}$ and although it is very weak $\left(0.002 e a_{0}\right)$, it is in the frequency range of the OROTRON. ${ }^{59}$ This instrument may be sensitive enough to observe this transition. ${ }^{60}$ The observation of this transition will give the first direct experimental determination of the acceptor tunneling splitting for $K=0$. To date only the sum of the $K=$ 0 and the $K=1$ splitting has been measured for $\left(\mathrm{H}_{2} \mathrm{O}\right)_{2}$, whereas for $\left(\mathrm{D}_{2} \mathrm{O}\right)_{2}$ the $K=0$ splitting was obtained under the assumption that it is not affected by a simultaneous $\mathrm{O}-\mathrm{D}$ stretch excitation. Another line that possibly may be seen is the parallel $K=1$ transition $E^{+} \rightarrow E^{-}$, which, although a hot band, is strong enough to be observable. 
To interpret the calculated transition dipole moments we introduced a group-theoretical model, and we separated the external part of a transition moment, associated with the wave functions describing the overall rotation of the dimer, from its internal part. The internal coordinates are the Euler angles of the monomers and the intermolecular distance. Both the external and the internal wave functions, as well as the external and internal part of the dipole operator, are of definite $G_{16}$ symmetry and obey specific selection rules with respect to this PI group. It could be shown that only states belonging to the twodimensional $E^{ \pm}$irreps of $G_{16}$ have a nonzero dipole expectation value, i.e., a permanent dipole moment. This value is close to the vector sum of the monomer dipoles plus induction effectscalculated at the equilibrium geometry of the dimer. The difference, a $10 \%$ increase, is due to vibrational averaging. For internal states belonging to the one-dimensional $A$ and $B$ irreps the expectation value of the dipole vanishes, but our model enabled us to relate the value of nonzero parallel transition moments associated with tunneling between these $A$ and $B$ states directly to the permanent dipole of the $E^{ \pm}$states.

We expect the transition moments also to be useful in the search for higher excited vibrational states. Knowledge of these states is important in the exploration of the energetically higher parts of the water pair potential. In particular, we found that the transition to the first excited stretch and the DT + AW combination band are both stronger than expected. The stretch transition was expected to be weak because the dipole moment function is only weakly dependent on $R$ and no strong coupling in the excited stretch state between $R$ and the Euler angles was envisioned. The transition to DT + AW is forbidden in the harmonic approximation. Finally, we recall that water dimers may absorb appreciably solar radiation in the atmosphere. ${ }^{38-43}$ The transition moments presented in this work might be useful in models describing this absorption.

Acknowledgment. This work was supported by the Chemistry Branch (Gebied Chemische Wetenschappen) of The Netherlands Organization for Scientific Research (NWO) and by NSF Grant No. CHE-9982134.

Supporting Information Available: Table 13S contains a complete list of the calculated vibrational transition dipole moments. This material is available free of charge via the Internet at http://pubs.acs.org.

\section{References and Notes}

(1) Rawls, R. Chem. Eng. News 2000, 78, 12.

(2) Malin, M. C.; Edgett, K. S. Science 2000, 288, 2330.

(3) Franks, F. Polywater; M. I. T. Press: Cambridge, Mass, 1981.

(4) Ball, P. Life's Matrix: A Biography of Water; Farrar and Straus and Giroux: New York, 1999.

(5) Stanley, H. E.; Buldyrev, S. V.; Canpolat, M.; Havlin, S.; Mishima, O.; Sadr-Lahijany, M. R.; Scala, A.; Starr, F. W. Physica D 1999, 133, 453.

(6) Luzar, A.; Chandler, D. Phys. Rev. Lett. 1996, 76, 928.

(7) Luzar, A.; Chandler, D. Nature 1996, 379, 55.

(8) Roberts, C. J.; Debenedetti, P. G.; Stillinger, F. H. J. Phys. Chem. $B$ 1999, 103, 10258 .

(9) Kistenmacher, H.; Lie, G. C.; Popkie, H.; Clementi, E. J. Chem. Phys. 1974, 61, 546.

(10) Chałasiński, G.; Szczęśniak, M. M.; Cieplak, P.; Scheiner, S. J. Chem. Phys. 1991, 94, 2873.

(11) van Duijneveldt-van de Rijdt, J. G. C. M.; van Duijneveldt, F. B. Chem. Phys. 1993, 175, 271.

(12) van Duijneveldt-van de Rijdt, J. G. C. M.; van Duijneveldt, F. B. Chem. Phys. Lett. 1995, 237, 560.

(13) Xantheas, S. S. J. Chem. Phys. 1994, 100, 7523.

(14) Klopper, W.; Schütz, M.; Lüthi, H.-P.; Leutwyler, S. J. Chem. Phys. 1995, 103, 1085.
(15) Hodges, M. P.; Stone, A. J.; Xantheas, S. S. J. Phys. Chem. A 1997, $101,9163$.

(16) Milet, A.; Moszynski, R.; Wormer, P. E. S.; van der Avoird, A. J. Phys. Chem. A 1999, 103, 6811-6819.

(17) Mahoney, M. W.; Jorgensen, W. L. J. Chem. Phys. 2000, 112, 8910.

(18) Mas, E. M.; Szalewicz, K.; Bukowski, R.; Jeziorski, B. J. Chem. Phys. 1997, 107, 4207.

(19) Mas, E. M.; Bukowski, R.; Szalewicz, K.; Groenenboom, G. C.; Wormer, P. E. S.; van der Avoird, A. J. Chem. Phys. 2000, 113, 6687.

(20) Groenenboom, G. C.; Mas, E. M.; Bukowski, R.; Szalewicz, K.; Wormer, P. E. S.; van der Avoird, A. Phys. Rev. Lett. 2000, 84, 4072.

(21) Groenenboom, G. C.; Wormer, P. E. S.; van der Avoird, A.; Mas, E. M.; Bukowski, R.; Szalewicz, K. J. Chem. Phys. 2000, 113, 6702.

(22) Dyke, T. R.; Mack, K. M.; Muenter, J. S. J. Chem. Phys. 1977, 66, 498.

(23) Fraser, G. T. Int. Rev. Phys. Chem. 1991, 10, 189.

(24) Zwart, E.; ter Meulen, J. J.; Meerts, W. L. Chem. Phys. Lett. 1990, 173,115 .

(25) Zwart, E.; ter Meulen, J. J.; Meerts, W. L.; Coudert, L. H. J. Mol. Spectrosc. 1991, 147, 27.

(26) Karyakin, E. N.; Fraser, G. T.; Suenram, R. D. Mol. Phys. 1993, $78,1179$.

(27) Karyakin, E. N.; Fraser, G. T.; Lovas, F. J.; Suenram, R. D.; Fujitake, M. J. Chem. Phys. 1995, 102, 1114.

(28) Busarow, K. L.; Cohen, R. C.; Blake, G. A.; Laughlin, K. B.; Lee, Y. T.; Saykally, R. J. J. Chem. Phys. 1989, 90, 3937.

(29) Pugliano, N.; Saykally, R. J. J. Chem. Phys. 1992, 96, 1832.

(30) Pugliano, N.; Cruzan, J. D.; Loeser, J. G.; Saykally, R. J. J. Chem. Phys. 1993, 98, 6600.

(31) Paul, J. B.; Provencal, R. A.; Saykally, R. J. J. Phys. Chem. A 1998, 102, 3279 .

(32) Braly, L. B. The intermolecular vibrations of the water dimer, Thesis, University of California at Berkeley, 1999.

(33) Braly, L. B.; Cruzan, J. D.; Liu, K.; Fellers, R. S.; Saykally, R. J. J. Chem. Phys. 2000, 112, 10293.

(34) Braly, L. B.; Liu, K.; Brown, M. G.; Keutsch, F. N.; Fellers, R. S.; Saykally, R. J. J. Chem. Phys. 2000, 112, 10314.

(35) Fellers, R. S.; Leforestier, C.; Braly, L. B.; Brown, M. G.; Saykally, R. J. Science 1999, 284, 945.

(36) Millot, C.; Stone, A. J. Mol. Phys. 1992, 77, 439

(37) Reimers, J. R.; Watts, R. O. Chem. Phys. 1984, 85, 83.

(38) Chýlek, P.; Geldart, D. J. W. Geophys. Res. Lett. 1997, 24, 2015.

(39) Tso, H. C. W.; Geldart, D. J. W.; Chýlek, P. J. Chem. Phys. 1998, $108,5319$.

(40) Chýlek, P.; Fu, Q.; Tso, H. C. W.; Geldart, D. J. W. Tellus A 1999, 51,304 .

(41) Daniel, J. S.; Solomon, S.; Sanders, R.; Portmann, R. W.; Miller,

D. C.; Madsen, W. J. Geophys. Res.-Atmos. 1999, 104, 16785.

(42) Evans, G. T.; Vaida, V. J. Chem. Phys. 2000, 113, 6652

(43) Goldman, N.; Fellers, R. S.; Leforestier, C.; Saykally, R. J. J. Phys Chem. A 2001, 105, 515.

(44) Biedenharn, L. C.; Louck, J. D. Angular Momentum in Quantum Physics; Encyclopedia of Mathematics; Addison-Wesley: Reading, Vol. 8, 1981.

(45) Messiah, A. Quantum Mechanics; volume 2 North-Holland: Amsterdam, 1969.

(46) van der Avoird, A.; Wormer, P. E. S.; Moszynski, R. Chem. Rev. 1994, 94, 1931.

(47) Colbert, D. T.; Miller, W. H. J. Chem. Phys. 1992, 96, 1982.

(48) Groenenboom, G. C.; Colbert, D. T. J. Chem. Phys. 1993, 99, 9681.

(49) Bunker, P. R.; Jensen, P. Molecular Symmetry and Spectroscopy; NRC Research Press: Ottawa, $2^{\text {nd }}$ ed.; 1998.

(50) Wormer, P. E. S.; Hettema, H. J. Chem. Phys. 1992, 97, 5592

(51) Wilson, E. B.; Decius, J. C.; Cross, P. C. Molecular Vibrations; McGraw-Hill: New York, 1955.

(52) Papousek, D.; Aliev, M. R. Molecular Vibrational-Rotational Spectra; Elsevier: Amsterdam, 1982.

(53) Wales, D. J. Rearrangements and Tunneling in Water Clusters. In Advances in Molecular Vibrations and Collision Dynamics; Bowman, J. M.; Bacic, Z., Eds.; JAI press: Stamford, 1998.

(54) Xantheas, S. S.; Dunning, T. H. J. Chem. Phys. 1993, 99, 8774.

(55) Herzberg, G. Molecular Spectra and Molecular Structure, Vol. 2: Infrared and Raman Spectra of Polyatomic Molecules; Van Nostrand: New York, 1945

(56) Dyke, T. R. J. Chem. Phys. 1977, 66, 492.

(57) Atkins, P. W.; Child, M. S.; Phillips, C. S. G. Tables for Group Theory; Oxford University Press: Oxford, 1970.

(58) van Bladel, J. W. I.; van der Avoird, A.; Wormer, P. E. S.; Saykally, R. J. J. Chem. Phys. 1992, 97, 4750.

(59) Roth, D. A.; Surin, L. A.; Dumesh, B. S.; Winnewisser, G.; Pak, I. J. Chem. Phys. 2000, 113, 3034.

(60) Pak, I. 2000, private communication. 